\title{
CO OBSERVATIONS OF THE INTERACTING GALAXY PAIR NGC 5394/95
}

\author{
Michele Kaufman, ${ }^{1}$ Kartik Sheth, ${ }^{2,3}$ Curtis Struck, ${ }^{4}$ Bruce G. Elmegreen, ${ }^{5}$ Magnus Thomasson, ${ }^{6}$ \\ Debra Meloy Elmegreen, ${ }^{7}$ and Elias Brinks ${ }^{8}$ \\ Received 2001 August 19; accepted 2001 October 29
}

\begin{abstract}
BIMA ${ }^{12} \mathrm{CO} J=1 \rightarrow 0$ observations are presented of the spiral galaxies NGC 5394 and NGC 5395 that have undergone a recent, grazing encounter. In NGC 5394 approximately $80 \%$ of the CO emission detected by BIMA is concentrated in the central $800 \mathrm{pc}$ (FWHM) starburst region, and the rest is from a portion of the inner disk south and west of the central starburst. In an encounter simulation that reproduces some of the main features of this galaxy pair, a considerable amount of gas in NGC 5394 falls into the central region early in the collision. The observed total gas distribution in the disk of NGC 5394 is lopsided, with more H I, CO, and $\mathrm{H} \alpha$ emission coming from the western or southwestern side. The innermost western arm of NGC 5394 is seen in $\mathrm{CO}$ and $\mathrm{H} \alpha$ emission, but the eastern inner-disk arm, which is very bright in the optical continuum, is not detected in $\mathrm{CO}$ or $\mathrm{H} \alpha$ emission. The NGC 5394 starburst region is similar in radio continuum luminosity and size to the M82 starburst and has a CO luminosity $~ 4$ times greater. A CO position-velocity diagram of the NGC 5394 nucleus reveals two separate velocity features very close to the center. This may indicate a nuclear ring or the "twin peaks" of an ILR or some depletion of ${ }^{12} \mathrm{CO} J=1 \rightarrow 0$ at the nucleus. From a comparison of the radio continuum, $\mathrm{H} \alpha, 60 \mu \mathrm{m}$, and CO luminosities, we estimate that the average extinction $A_{v}$ of the starburst nucleus is 3-4 mag, the star formation rate is $\sim 6 M_{\odot} \mathrm{yr}^{-1}$, and the conversion factor $N\left(\mathrm{H}_{2}\right) / I_{\mathrm{CO}}$ in the starburst is a factor of 3-4 below the standard value. Comparison of NGC 5394 with two other systems previously studied suggests that in prograde grazing encounters a central starburst may not develop until near the end of the ocular phase. Very little of the CO emission from NGC 5395 found in previous single-dish observations is detected in the BIMA data; thus molecular gas in NGC 5395 does not appear to be strongly concentrated in compressed ridges.
\end{abstract}

Key words: galaxies: individual (NGC 5394/95) — galaxies: interactions — galaxies: ISM — galaxies: starburst

\section{INTRODUCTION}

The interacting spiral galaxies NGC 5394/95 (Arp 84) at a distance of $47 \mathrm{Mpc}$ have recently undergone a nearly grazing encounter. Figure 1 displays an $r$-band image of the system from the Chengalur-Nordgren galaxy survey (Nordgren et al. 1997). From a comparison of the observed tidal features of the system with galaxy encounter simulations Kaufman et al. (1999a, hereafter Paper I) concluded that NGC 5394, the smaller member of the pair, suffered a close prograde collision with NGC 5395 and is in an immediate post-ocular (posteye-shaped) phase. NGC 5394 has a central starburst, 3.5 (=800 pc) in diameter, very bright inner-disk spiral

\footnotetext{
${ }^{1}$ Department of Physics and Department of Astronomy, Ohio State University, 174 West 18th Avenue, Columbus, OH 43210; rallis@ mps.ohio-state.edu.

${ }^{2}$ Department of Astronomy, University of Maryland, College Park, MD 20741-2421.

${ }^{3}$ Current address: Division of Mathematical and Physical Sciences, California Institute of Technology, MS 105-24, Pasadena, CA 91125; kartik@astro.caltech.edu.

${ }^{4}$ Department of Physics and Astronomy, Iowa State University, Ames, IA 50010; curt@iastate.edu.

${ }^{5}$ IBM Research Division, T. J. Watson Research Center, P.O. Box 218, Yorktown Heights, NY 10598; bge@watson.ibm.com.

${ }^{6}$ Onsala Space Observatory, SE-439 92 Onsala, Sweden; magnus@ oso.chalmers.se.

${ }^{7}$ Department of Physics and Astronomy, Vassar College, Poughkeepsie, NY 12604; elmegreen@vassar.edu.

8 Departamento de Astronomía, Universidad de Guanajuato, Apdo. Postal 144, Guanajuato, Gto. 36000, Mexico; ebrinks@astro.ugto.mx.
}

arms $\left(R=4^{\prime \prime}-13^{\prime \prime}=0.9-3.0 \mathrm{kpc}\right.$ from the nucleus $)$, and two long, open tidal arms, with the tidal tail extending to $R=80^{\prime \prime}$. Its disk is intrinsically oval. Some properties of NGC 5394 are listed in Table 1. The dominant spiral arm of its companion, NGC 5395, forms a large ring or pseudoring of $\mathrm{H} \alpha$, radio continuum, and $\mathrm{H}$ I emission. The eastern half of the ring is the brighter side in radio continuum and $\mathrm{H} \alpha$, whereas the western half is the brighter side in $\mathrm{H}$ I.

NGC 5394/95 is one of three galaxy pairs that we have studied previously with radio, optical, and singledish $\mathrm{CO}$ observations and numerical simulations to see how structures resulting from prograde, in-plane, grazing encounters develop. The other two systems are IC 2163/ NGC 2207 (Elmegreen et al. 1995a, 1995b, 2000, 2001) and NGC 2535/36 (Kaufman et al. 1997). In the models the response of the galaxy disk to the $\cos 2 \theta$ tidal potential of the prograde encounter produces an eyeshaped (ocular) oval like that seen in IC 2163 and NGC 2535. This can subsequently evolve into inner-disk arms, similar to those visible in NGC 5394. From a comparison of the observed morphologies with the models Paper I concluded that the galaxies IC 2163, NGC 2535, and NGC 5394 form an "age" sequence, with IC 2163 the least evolved interaction and NGC 5394 the most evolved one. IC 2163 has star formation typical of normal spiral disks (Elmegreen et al. 2001); NGC 2535 has somewhat enhanced star formation (Bernlöhr 1993), with the brightest $\mathrm{H} \alpha$ emission at the two ends of the eye-shaped oval (Amram et al. 1989); and NGC 5394 has a central starburst. 


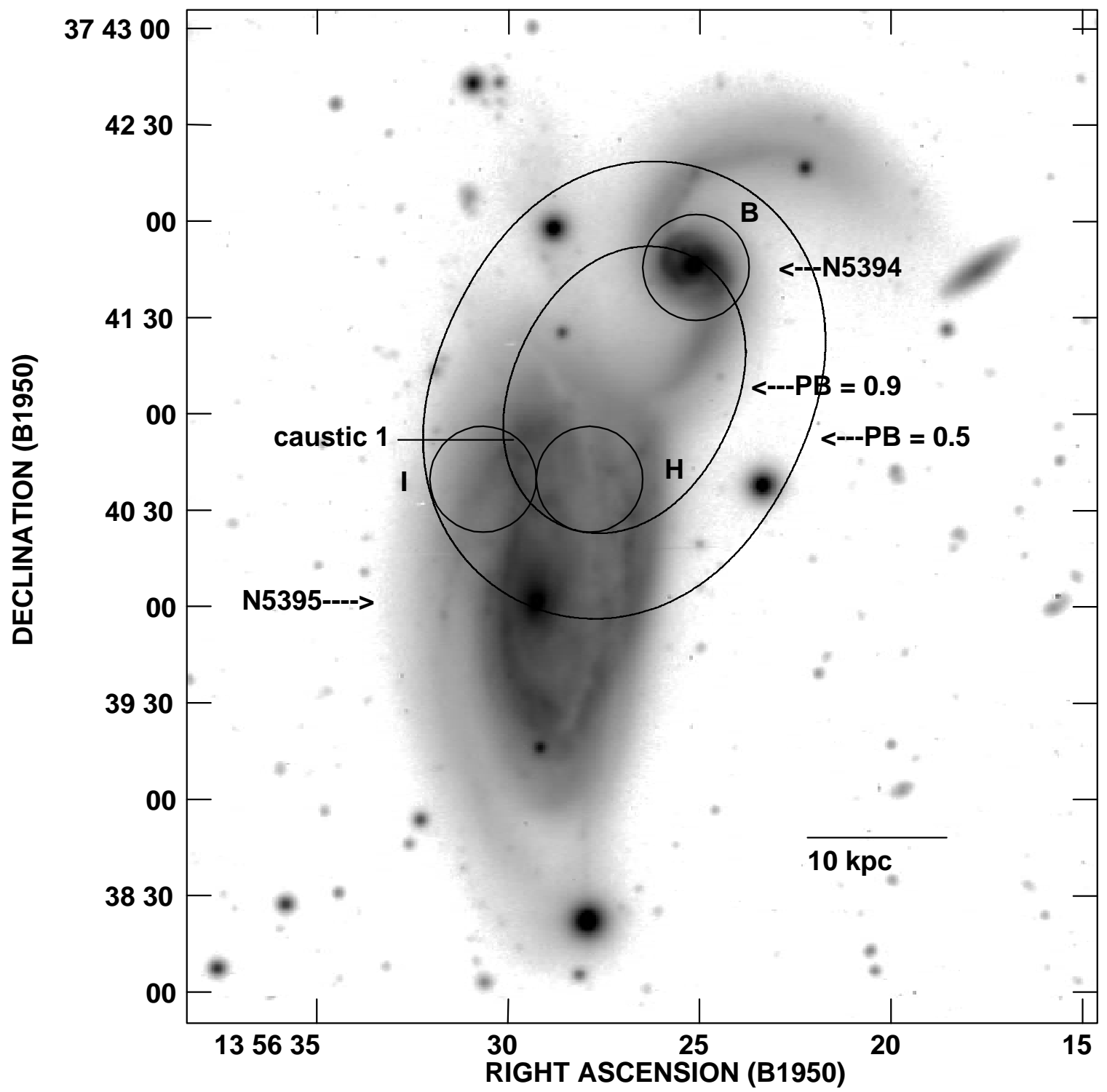

FIG. 1.- The $r$-band image of NGC 5394/95 (on a logarithmic intensity scale) from the Chengalur-Nordgren galaxy survey. The circles labelled "B," "H," and "I" denote the Onsala HPBW at the positions where CO emission was clearly detected by Onsala Space Observatory. Position B contains the bright inner-disk arms and starburst nucleus of NGC 5394. The field observed in ${ }^{12} \mathrm{CO} J=1 \rightarrow 0$ in the BIMA observations presented here is indicated by the two oval contours marked "PB," which represent the $50 \%$ and the $90 \%$ sensitivity contours of the combined primary beams after the two pointings were mosaicked. In NGC 5394 all of the CO emission clearly detected in the BIMA data is from the central and southwestern portion of position B. The stellar feature in NGC 5395 labelled "caustic 1" was interpreted in Paper I as a caustic produced by temporary convergence of orbits.

We present ${ }^{12} \mathrm{CO} J=1 \rightarrow 0$ observations of NGC 5394/ 95 taken with the Berkeley-Illinois-Maryland Association (BIMA) $)^{9}$ array to examine the distribution of molecular gas, particularly in the inner-disk arms and starburst nucleus of NGC 5394 and in the ring/pseudoring of NGC 5395.

The inner-disk arms of NGC 5394 present a puzzle for models of star formation. These bright arms are mostly free of star formation knots (Arp 1969; Wray 1988), and only one of the three arms is clearly detected in $\mathrm{H} \alpha$ (Paper I). This is unusual, since high surface brightness in a spiral arm is almost always the result of ongoing star formation. Wray (1988) described these arms as extremely bright spiral arcs

${ }^{9}$ The BIMA array is partially funded by grant AST 99-81289 from the National Science Foundation that are "mostly smooth and green." Despite the presence of $\mathrm{H}$ I gas, two of these arms show no evidence of star formation in progress. Paper I offered the following interpretation. If molecular gas can be ignored, the instability parameter $Q_{\text {gas }}>10$, which is too high for significant star formation; Kennicutt (1989) and Martin \& Kennicutt (2001) found that $Q_{\text {gas }} \leq 1.4$ for star-forming regions in late-type spirals. A value for $Q_{\text {gas }}$ based simply on $\mathrm{H}$ I may be too high if a significant amount of molecular gas, which can lower the value of $Q_{\text {gas }}$, is present. Thus we needed to measure the molecular surface density at the inner-disk arms of NGC 5394. Single-dish observations with the NRAO $12 \mathrm{~m}$ telescope at a resolution of 55" (Zhu et al. 1999) and with the Onsala Space Observatory at a resolution of 33" (Paper I) detected significant CO emission from NGC 5394. In particular, the Onsala observations found a lot of ${ }^{12} \mathrm{CO} J=1 \rightarrow 0$ emission (129 $\mathrm{Jy} \mathrm{km} \mathrm{s}^{-1}$ ) in the field 
TABLE 1

PRoperties OF NGC 5394

\begin{tabular}{|c|c|c|}
\hline Parameter & Value & Reference \\
\hline 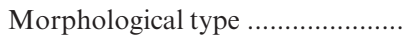 & $\mathrm{SB}(\mathrm{s}) \mathrm{b}$ pec & 1 \\
\hline \multicolumn{3}{|l|}{ Radio continuum nucleus: } \\
\hline Right ascension (1950.0)........... & $13^{\mathrm{h}} 56^{\mathrm{m}} 25^{\mathrm{s}} .10$ & 1 \\
\hline Declination (1950.0) ....................... & $37^{\circ} 41^{\prime} 45^{\prime \prime} 7$ & 1 \\
\hline Isophotal major radius $R_{25} \ldots \ldots \ldots \ldots$ & $51^{\prime \prime}$ & 1 \\
\hline$B_{T}$ & 13.70 & 1 \\
\hline 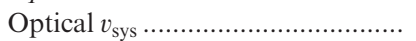 & $3451 \pm 12 \mathrm{~km} \mathrm{~s}^{-1}$ & 1 \\
\hline 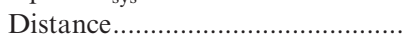 & $47 \mathrm{Mpc}$ & 2 \\
\hline Linear scale & $230 \mathrm{pc} \operatorname{arcsec}^{-1}$ & 2 \\
\hline Disk inclination & $15^{\circ} \pm 2^{\circ}$ & 6 \\
\hline Projection line of nodes .................... & $0^{\circ} \pm 2^{\circ}$ & 2 \\
\hline 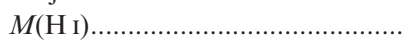 & $7.3 \times 10^{8} M_{\odot}$ & 2 \\
\hline 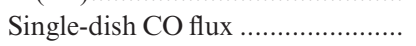 & $168 \mathrm{Jy} \mathrm{km} \mathrm{s}^{-1}$ & 2 \\
\hline Semimajor axis of primary bar ..... & $8^{\prime \prime}$ & 2 \\
\hline Position angle of bar major axis ... & $120^{\circ}$ & 2 \\
\hline 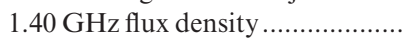 & $34.8 \mathrm{mJy}$ & 2 \\
\hline 4.86 GHz flux density ....................... & $15 \mathrm{mJy}$ & 3 \\
\hline \multicolumn{3}{|l|}{ Central starburst: } \\
\hline $1.45 \mathrm{GHz}$ flux density ................. & $29 \mathrm{mJy}$ & 4 \\
\hline 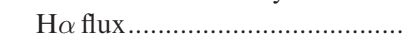 & $2.2 \times 10^{-13} \mathrm{ergs} \mathrm{cm}^{-2} \mathrm{~s}^{-1}$ & 5 \\
\hline
\end{tabular}

REFERENCES.-(1) de Vaucouleurs et al. 1991 and the NASA Extragalactic Database; (2) Kaufman et al. 1999a; (3) Condon, Frayer, \& Broderick 1991; (4) Becker, White, \& Helfand 1995; (5) Keel et al. 1985; (6) this paper

containing the starburst nucleus and the inner-disk spiral arms, but they did not have sufficient spatial resolution to distinguish between emission from the nuclear region and that from the inner-disk arms (Paper I). The 5 times higher resolution $(\sim 6$ ". 5$)$ of the BIMA ${ }^{12} \mathrm{CO} J=1 \rightarrow 0$ observations presented here allows us to make this distinction.

Optical spectroscopy by Arp (1969) and Leech et al. (1989) and near-infrared spectroscopy by Vanzi, AlonsoHerrero, \& Rieke (1998) revealed a young starburst in the center in NGC 5394. The position of NGC 5394 in the nearinfrared line-ratio diagnostic diagrams indicates a starburst of duration a few megayears, observed several megayears after the peak star-formation episode. The starburst in NGC 5394 has nearly the same radio continuum luminosity and linear size as the central starburst in M82 (Paper I; Condon et al. 1990). Unlike M82, NGC 5394 is viewed nearly face-on. The $\mathrm{H} \alpha$ line profile at the nucleus of NGC 5394 has a $100 \mathrm{~km} \mathrm{~s}^{-1}$ velocity asymmetry (an extended blue wing), which may represent a wind/outflow of ionized gas perpendicular to the disk (Paper I). In the M82 starburst an outflow speed of several hundred kilometers per second is seen in the molecular gas (Shen \& Lo 1995) as well as in the ionized gas. Our BIMA observations of NGC 5394, particularly the velocity data, provide information about the structure of the starburst region in NGC 5394 and the relative spatial distributions of ${ }^{12} \mathrm{CO} \quad J=1 \rightarrow 0$ and $\mathrm{H} \alpha$ emission.

For the "standard" value of the Milky Way conversion factor $X=N\left(\mathrm{H}_{2}\right) / I_{\mathrm{CO}}$, we adopt $2.3 \pm 0.3 \times 10^{20} \mathrm{H}_{2} \mathrm{~cm}^{-2}$ $\left(\mathrm{K} \mathrm{km} \mathrm{s}^{-1}\right)^{-1}$ from Strong et al. (1988) since this value has been widely used. More recent determinations for the Milky Way give somewhat lower values of $1.9 \pm 0.2 \times 10^{20}$ (Strong \& Mattox 1996) and $1.8 \pm 0.3 \times 10^{20}$ (Dame, Hartmann, \& Thaddeus 2001). We shall estimate the value of $X$ for the starburst region of NGC 5394 by comparing its radio continuum, $\mathrm{H} \alpha, 60 \mu \mathrm{m}$, and $\mathrm{CO}$ luminosities. For luminous infrared galaxies involved in mergers, measurements of the dynamical mass (Alonso-Herrero et al. 2001; Shier, Rieke, \& Rieke 1994) and estimates of the molecular mass based on millimeter-wave thermal emission of dust (Braine \& Dumke 1998 ) indicate that the standard value of $X$ overestimates the molecular mass by factors ranging from 3 to greater than 10 in these powerful starbursts. The starburst in NGC 5394 is more modest, comparable to that in M82. Wild et al. (1992) and Smith et al. (1991) found that the value of $X$ in the M82 starburst is a factor of 3 below the standard value.

The northern half of NGC 5395 was included as well in our BIMA observations. ${ }^{12} \mathrm{CO} J=1 \rightarrow 0$ emission from NGC 5395 was detected in the Onsala observations (Paper I) and in the NRAO $12 \mathrm{~m}$ observations by Zhu et al. (1999). In other galaxies nonthermal radio continuum emission and ${ }^{12} \mathrm{CO} J=1 \rightarrow 0$ emission are usually correlated on scales of $\sim 2 \mathrm{kpc}$ (Adler, Allen, \& Lo 1991). Since the eastern half of the ring/pseudoring in NGC 5395 is the brighter side in radio continuum emission but the fainter side in $\mathrm{H} \mathrm{I}$, we wanted to find out whether the eastern half of the ring is the brighter side in $\mathrm{CO}$ emission. We expected to see a strong shock front in the molecular gas along the radio continuum ridge. We also looked for any $\mathrm{CO}$ emission associated with an unusual "shell-like" stellar structure on the northeastern side (marked as "caustic 1" in Fig. 1), which was interpreted in Paper I as a caustic produced by temporary convergence of orbits.

In addition to the BIMA $\mathrm{CO}$ data, our analysis uses $\mathrm{H} \mathrm{I}$, Fabry-Perot $\mathrm{H} \alpha$, radio continuum, single-dish $\mathrm{CO}$, and broadband near-infrared and optical observations from Paper I and the VLA radio continuum image from the FIRST survey (Becker, White, \& Helfand 1995).

With the adopted distance of $47 \mathrm{Mpc}$ for the galaxy pair, $1^{\prime \prime}$ corresponds to $230 \mathrm{pc}$. The velocities in this paper are 
heliocentric and use the optical definition for the nonrelativistic Doppler shift. ${ }^{10}$

Section 2 describes the observations and the data reduction. Section 3 describes the optical and near-infrared morphology of NGC 5394. Sections 4, 5, 6, and 7 present our CO results on NGC 5394, with the general CO distribution in $\S 4$, the eastern inner-disk arm in $\S 5$, the central starburst in $\S 6$, and a comparison of $\mathrm{CO}, \mathrm{H} \alpha$, and $\mathrm{H}$ I velocity fields in $\S 7$. Section 8 discusses the implications for development of a starburst in a nonmerging encounter of this type. Section 9 discusses NGC 5395. Section 10 contains our conclusions.

\section{OBSERVATIONS AND DATA REDUCTION}

The BIMA ${ }^{12} \mathrm{CO} J=1 \rightarrow 0$ observations were made on 1999 June 4 with 10 telescopes in C configuration. We used two pointings to cover NGC 5394 and the northern half of NGC 5395. The duration of the run was $10.7 \mathrm{hr}$. Table 2 gives a summary of the observations. The phase calibrator was the quasar $1310+323$, the bandpass calibrator was $3 \mathrm{C}$ 273, and the absolute flux standard was Mars. The observations have a velocity resolution of $4.06 \mathrm{~km} \mathrm{~s}^{-1}$ and were centered on $3497 \mathrm{~km} \mathrm{~s}^{-1}$, with a total bandwidth of $908 \mathrm{~km} \mathrm{~s}^{-1}$.

The data reduction was carried out using standard reduction algorithms from the MIRIAD package (Sault, Teuben, $\&$ Wright 1995). The data were Hanning smoothed to $10 \mathrm{~km}$ $\mathrm{s}^{-1}$ channels. The two pointings were mosaicked to form a single cube with pixel size $=1.15$, synthesized beam $=6 "$ " $6 \times 6 "$ " 3 , and dimensions $198 \times 211 \times 60$ pixels (R.A. $\times$ decl. $\times$ velocity), covering the velocity range 3208 to $3808 \mathrm{~km} \mathrm{~s}^{-1}$. The dirty channel maps were then deconvolved by using the Steer-Dewdney-Ito CLEAN algorithm (Steer, Dewdney, \& Ito 1984). The rms noise in the cleaned $10 \mathrm{~km} \mathrm{~s}^{-1}$ channel maps is $80 \mathrm{mJy}$ beam ${ }^{-1}$, only slightly higher than the theoretical noise of $76 \mathrm{mJy}^{-1}$ beam ${ }^{-1}$. In Figure 1 the $50 \%$ and $90 \%$ sensitivity contours of the combined primary beams after mosaicking the data are overlaid on the $r$-band image in gray scale. Figure 2 displays channel maps from the BIMA CO cube overlaid on a gray-scale display of the Digitized Sky Survey image ${ }^{11}$ for the range of velocities appropriate to NGC 5394. Moment maps of the total emission and of the intensity-weighted velocity field were made from this cube.

Areas of genuine $\mathrm{CO}$ emission were selected by clipping at 2 times the rms noise in a cube convolved to $12^{\prime \prime}$ resolution and requiring that a feature be continuous in velocity in at least two adjacent channels. In NGC 5394 the only ${ }^{12} \mathrm{CO}$ $J=1 \rightarrow 0$ emission seen in the BIMA observations is from the central and southwestern half of the Onsala position B marked in Figure 1, that is, only from the central part of the galaxy. The CO emission from NGC 5394 is present over the velocity range 3388 to $3528 \mathrm{~km} \mathrm{~s}^{-1}$, and the peak brightness temperature $T_{b}$ in the channel maps is $2.05 \mathrm{~K}$ (after correction for primary beam attenuation). BIMA finds very little CO emission from NGC 5395; a small region inside

\footnotetext{
${ }^{10}$ Where necessary, the relation $v_{\text {helio,opt }}=\mathrm{v}_{\text {lsr,radio }}+28 \mathrm{~km} \mathrm{~s}^{-1}$ was used to convert velocities to the heliocentric, optical system. Recall that the optical definition is $v_{\mathrm{opt}}=c\left(\lambda-\lambda_{0}\right) / \lambda_{0}$, where $\lambda_{0}$ is the rest wavelength.

11 The POSS Digitized Sky Survey was produced at the Space Telescope Science Institute under grant NAG W-2166.
}

TABLE 2

SUMMARY OF BIMA OBSERVATIONS

\begin{tabular}{|c|c|}
\hline Parameter & Value \\
\hline Date of observation ....... & 1999 Jun 4 \\
\hline 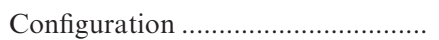 & $\mathrm{C}$ \\
\hline Synthesized beam (HPBW, P.A.) .... & $6^{\prime \prime} .6 \times 6^{\prime \prime} .3,86^{\circ}$ \\
\hline \multicolumn{2}{|l|}{ Pointing centers: } \\
\hline 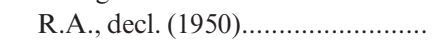 & $13^{\mathrm{h}} 56^{\mathrm{m}} 26^{\mathrm{s}} .152,+37^{\circ} 41^{\prime} 27^{\prime \prime} .14$ \\
\hline 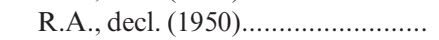 & $13^{\mathrm{h}} 56^{\mathrm{m}} 27^{\mathrm{s}} .797,+37^{\circ} 40^{\prime} 47^{\prime \prime} \cdot 21$ \\
\hline 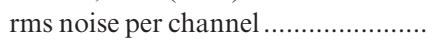 & $80 \mathrm{mJy}_{\text {beam }}{ }^{-1}=0.18 \mathrm{~K}$ \\
\hline Equivalent $T_{b}$ for $1 \mathrm{Jy}$ beam $^{-1} \ldots \ldots \ldots$ & $2.21 \mathrm{~K}$ \\
\hline 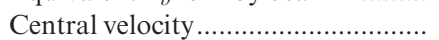 & $3497 \mathrm{~km} \mathrm{~s}^{-1}$ \\
\hline Velocity resolution & $4.06 \mathrm{~km} \mathrm{~s}^{-1}$ \\
\hline Hanning-smoothed channel width .. & $10 \mathrm{~km} \mathrm{~s}^{-1}$ \\
\hline 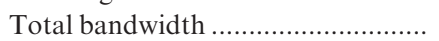 & $908 \mathrm{~km} \mathrm{~s}^{-1}$ \\
\hline 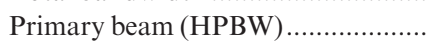 & $100^{\prime \prime}$ \\
\hline Largest scale size visible to array ...... & $40^{\prime \prime}$ \\
\hline
\end{tabular}

the arc formed by the stellar caustic has a peak $T_{b}$ of $0.87 \mathrm{~K}$ $(=4.8$ times the rms noise $)$ at $v=3308 \mathrm{~km} \mathrm{~s}^{-1}$.

\section{OPTICAL AND INFRARED MORPHOLOGY OF NGC 5394}

NGC 5394 has $M_{B}=-19.7$. On the basis of the H I velocity field the observed value of $v_{\max } \sin i$ and the $I$-band luminosity, Paper I concluded that NGC 5394 is viewed nearly face-on, with its major axis of projection into the sky plane at a position angle of $0^{\circ}$. The stellar disk of NGC 5394 is intrinsically oval as a result of the encounter. In the sky plane the position angle of the photometric major axis varies continuously from $60^{\circ}$ to $-6^{\circ}$, and the ellipticity increases from 0.21 to 0.60 (Paper I) as the galactocentric distance increases from 13" (the start of the tidal arms) to $51^{\prime \prime}$ (the isophotal major radius $R_{25}$ measured by de Vaucouleurs et al. 1991). Ignoring the small difference at $R_{25}$ between the position angles of the photometric major axis and the projection line of nodes, the area of the face-on disk at the 25th magnitude isophote $=\pi a_{0} b_{0}=\pi(b \sec i / a) R_{25}^{2}$, where $a, b$ are the semimajor and semiminor axes in the sky plane and $a_{0}, b_{0}$ are the face-on values. For comparisons with other galaxies, as in $\S 8$ below, we take the effective value of $R_{25}$ as $\left(R_{25}\right)_{\mathrm{eff}}=(b \sec i / a)^{1 / 2} R_{25}$. With inclination $i=15^{\circ}$ and $b / a=0.4$, this gives $\left(R_{25}\right)_{\mathrm{eff}}=33^{\prime \prime}=7.6 \mathrm{kpc}$.

NGC 5394 has two long, open tidal arms (see Fig. 1) with high arm-interarm contrast (Paper I). The very bright innerdisk spiral arms are disjoint from the long outer tidal arms, as the latter depart from the side of the inner-disk arms at a large angle. The left panel of Figure 3 is a sketch of features visible in the inner disk and center of NGC 5394. The eastern inner-disk arm spirals outward for $210^{\circ}$ in a clockwise direction, starting at $R=4^{\prime \prime}$ at a position angle of $200^{\circ}$ and ending at $R=13^{\prime \prime}$. The western inner-disk arm spirals outward from $R=4^{\prime \prime}$ at a position angle of $30^{\circ}$ and appears to bifurcate at a position angle of $-50^{\circ}$ into what we call the two western inner-disk arms. The outer of the two western inner-disk arms is symmetric to the eastern inner-disk arm, whereas the innermost western arm seems to be a separate structure. There is no evidence of ongoing star formation in either the eastern or the outer of the two western inner-disk arms. $\mathrm{H} \alpha$ emission is detected from the innermost western arm (Paper I). 


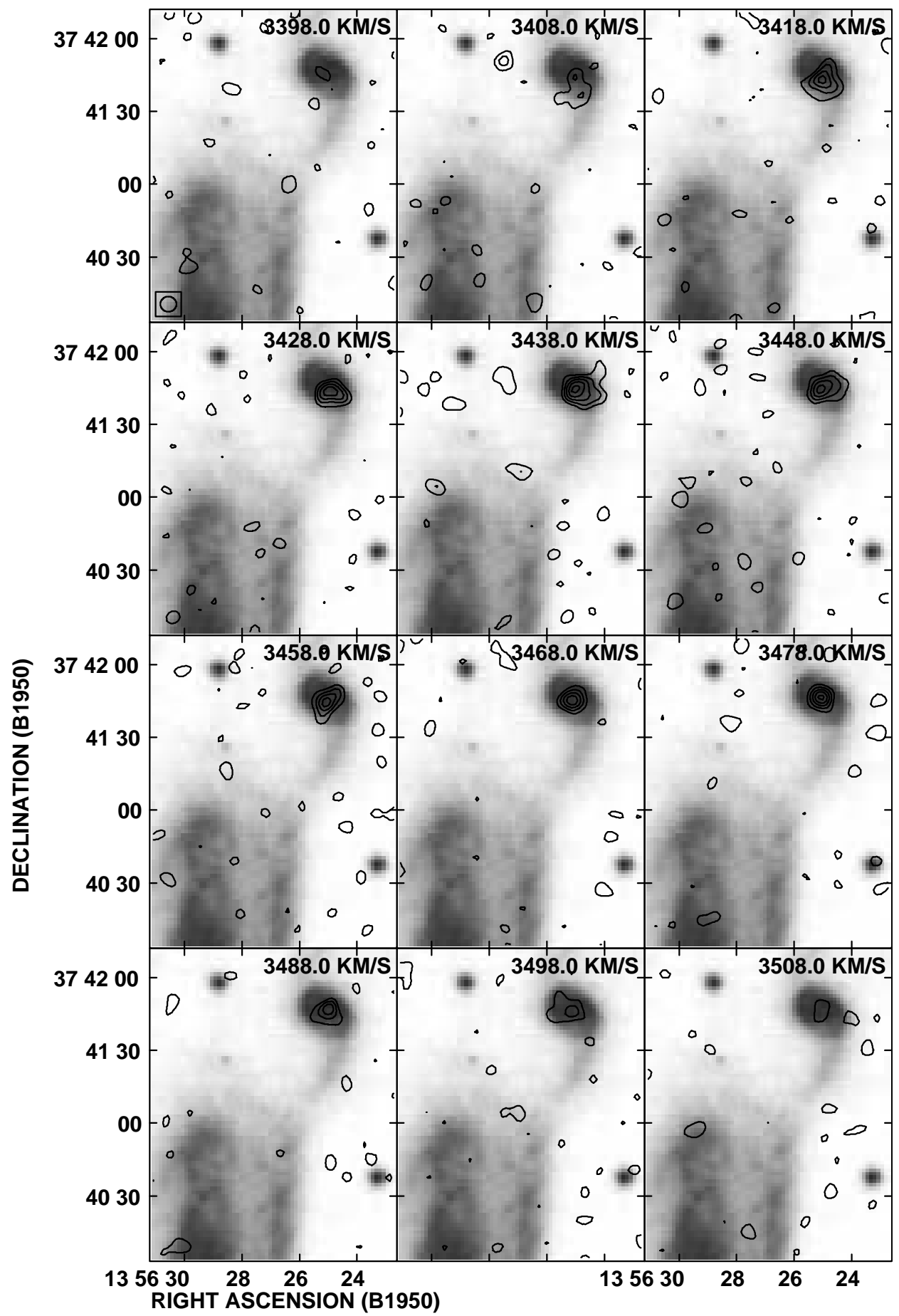

FIG. 2.-Channels maps from the BIMA CO cube overlaid as contours on a gray-scale display of the Digitized Sky Survey image for the range of velocities appropriate to NGC 5394. The contour levels are 2, 4, 6, 8, and 10 times the rms noise of $0.18 \mathrm{~K}$.

There is a barlike structure (see Fig. 3, left) with a major axis of $16^{\prime \prime}(=3.7 \mathrm{kpc})$ at a position angle of $120^{\circ}$ (Paper I; Bushouse \& Stanford 1992). We shall refer to this feature as the primary bar; with an axis ratio of $\sim 1.7$, it is a fat bar. The inner-disk arms appear tangent to part of the primary bar but do not depart quite from the ends of the bar. In the optical images, such as the Chengalur-Nordgren $r$-band image (Nordgren et al. 1997) in Figure 1, which has 2".3 resolution, one does not see straight dust lanes along the primary bar. Straight dust lanes are the signature of a bar that extends between an inner Lindblad resonance and corota- tion (Athanassoula 1992a, 1992b). Measured widths of dust lanes in galaxies with large bars are, typically, 100-250 pc (Benedict 1982) on NGC 7479 and our measurements on NGC 1300 and NGC 5236 (from images in Elmegreen et al. 1996 and Sandage \& Bedke 1988, respectively). At the distance of NGC 5394 this corresponds to 0 ". $5-1$ ". 0 , so, if present in NGC 5394, the optical resolution may not be good enough to see them. Interior to the primary bar the near-infrared $J$ and $K$-band images by Bushouse \& Stanford (1992) reveal an elongated feature with a major axis of $\sim 7^{\prime \prime}$ and a position angle of $90^{\circ}$, thus trailing the bar by $30^{\circ}$ in 

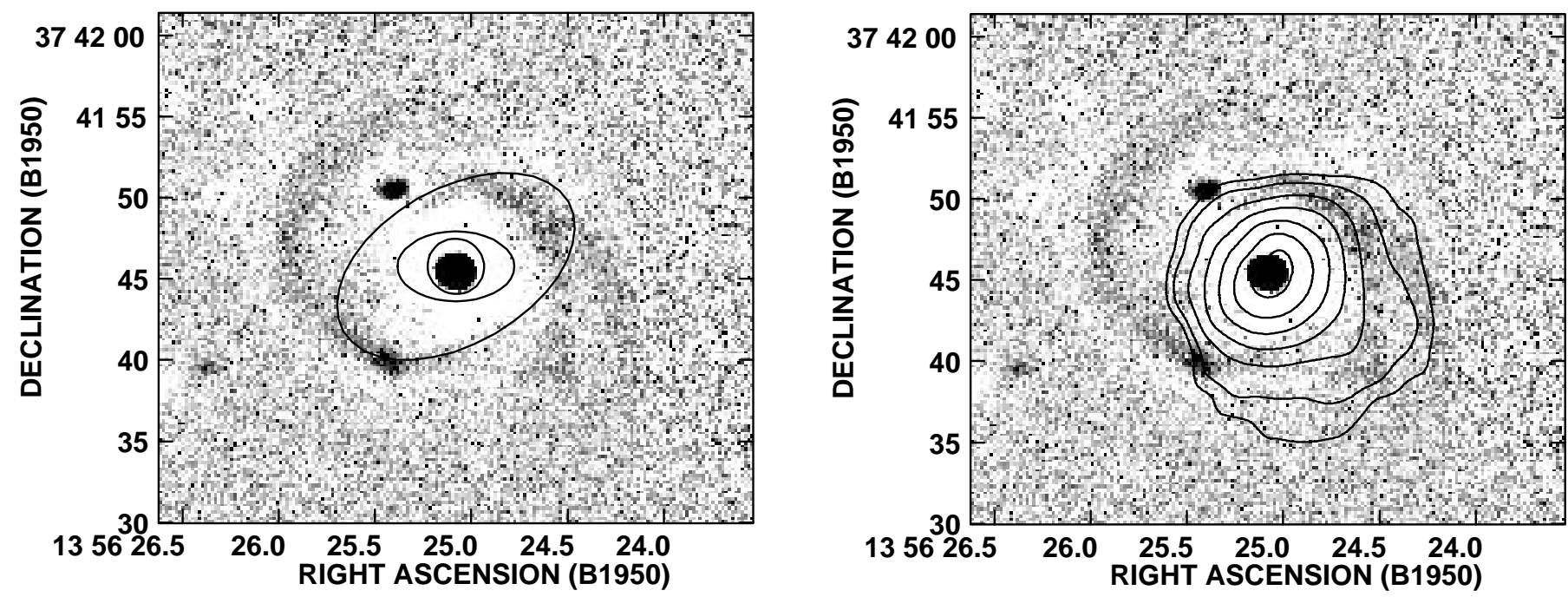

FIG. 3.-Left: Sketch of features visible in optical and near-infrared images of the center of NGC 5394. An unsharp-masked $J$-band image, which shows the inner-disk arms, is displayed in gray scale. The western inner-disk arm appears to bifurcate at a position angle of $-50^{\circ}$ into what we call the two western innerdisk arms. The larger of the two oval contours represents the primary barlike feature with a semimajor axis of $8^{\prime \prime}$; the smaller oval contour is a possible secondary bar with a semimajor axis of 3".5. The central circle indicates the extent of the starburst. Right: Contours of BIMA CO integrated intensity, after correction for primary-beam attenuation, overlaid on the unsharp-masked $J$-band image in gray scale. The $I_{\mathrm{CO}}$ contours are at 5, 10, 20, 30, 40, 50, 60 Jy beam ${ }^{-1} \mathrm{~km}$ $\mathrm{s}^{-1}$, where $10 \mathrm{Jy}_{\text {beam }}^{-1} \mathrm{~km} \mathrm{~s}^{-1}=22 \mathrm{~K} \mathrm{~km} \mathrm{~s}^{-1}$, and the rms noise is $2.8 \mathrm{Jy} \mathrm{beam}^{-1} \mathrm{~km} \mathrm{~s}^{-1}$. At this location, the correction factor for primary-beam attenuation in the mosaicked BIMA data is 1.08. No CO emission is detected from the eastern inner-disk arm, which is bright in the optical continuum.

azimuth. This smaller oval feature is not evident in the $J$ band image ( $1^{\prime \prime}$ resolution) in Paper I or the 2-MASS $J, H$, $K$ images (3".3 resolution) or in the Chengalur-Nordgren $r$ band image. In $\S 7$ we consider the kinematic evidence for this possible secondary bar.

From the twist of the $\mathrm{H}$ I velocity contours at the outer tidal arm, Paper I concluded that the outer tidal arms are outside of corotation. If the outer arms are driving a density wave in the inner arms (it is not clear that this is occurring), then the radius where the inner-disk arms end and the outer tidal arms begin (1.6 times the semimajor axis of the primary bar) may represent either corotation or the outer Lindblad resonance for the inner-disk spiral arms.

\section{GENERAL CO DISTRIBUTION IN NGC 5394}

The Onsala observations (Paper I) measured a total integrated ${ }^{12} \mathrm{CO} J=1 \rightarrow 0$ flux $S_{\mathrm{CO}}$ of $168 \mathrm{Jy} \mathrm{km} \mathrm{s}{ }^{-1}$ from a five-position survey of NGC 5394 with a 33" (HPBW) beam at $33^{\prime \prime}$ spacing; of this, $129 \mathrm{Jy} \mathrm{km} \mathrm{s}^{-1}$ is from the pointing (position B in Fig. 1) centered on the starburst nucleus and the rest is from a weak detection (3 times rms noise) in a pointing centered $33^{\prime \prime}$ south of the nucleus, which includes the southern tidal arm. With the NRAO $12 \mathrm{~m}$ telescope Zhu et al. (1999) measured a CO integrated flux of $154 \mathrm{Jy} \mathrm{km} \mathrm{s}^{-1}$ in a 55" (HPBW) beam; this is consistent with the Onsala value. With the adopted standard value of the conversion factor $X$, the Onsala $S_{\mathrm{CO}}$ corresponds to an $M\left(\mathrm{H}_{2}\right)$ of $3.4 \times 10^{9} M_{\odot}$. Paper I found $M(\mathrm{H} \mathrm{I})=7.3 \times 10^{8} M_{\odot}$. After adding a $36 \%$ by mass contribution from helium to the atomic plus molecular hydrogen mass, this gives a total mass in gas of $5.5 \times 10^{9} M_{\odot}$. However, as mentioned in $\S 1$, use of the standard value of $X$ in a starburst region is questionable. We shall estimate $X$ for the starburst region in $\S 6$ and discuss the $M\left(\mathrm{H}_{2}\right) / M\left(\mathrm{H}_{\mathrm{I}}\right)$ ratio for the galaxy in $\S 8$.

In the right panel of Figure 3 contours of the BIMA ${ }^{12} \mathrm{CO}$ $J=1 \rightarrow 0$ integrated-intensity $I_{\mathrm{CO}}$, after correction for pri- mary-beam attenuation, are overlaid on an unsharpmasked $J$-band image ( $1^{\prime \prime}$ resolution, from Paper I) in gray scale to show the location of the $\mathrm{CO}$ emission relative to the eastern inner-disk arm and the two western inner-disk arms. The right panel of Figure 4 displays contours of $\mathrm{H} \alpha$ emission (2".4 resolution, from Paper I) overlaid on the $I_{\mathrm{CO}}$ image in gray scale. In the nucleus there is a marginal displacement between the surface brightness maximum in $\mathrm{CO}$ and the optical/radio continuum surface brightness maximum (see $\S 6$ below). Aside from this, the ${ }^{12} \mathrm{CO} J=1 \rightarrow 0$ emission and $\mathrm{H} \alpha$ emission have a similar lopsided distribution of extended emission toward the southwest. In particular, no $\mathrm{CO}$ or $\mathrm{H} \alpha$ emission is detected from the eastern inner-disk arm, which is bright in the optical continuum. Star formation in the disk appears to follow the distribution of molecular gas.

The total integrated $\mathrm{CO}$ flux detected with BIMA is $S_{\mathrm{CO}}=117 \mathrm{Jy} \mathrm{km} \mathrm{s}^{-1}$, with an rms noise of $\pm 8 \mathrm{Jy} \mathrm{km} \mathrm{s}^{-1}$ and an uncertainty in the flux calibration of $10 \%-15 \%$. For the aperture containing the starburst nucleus and the inner disk arms (position B), the single-dish Onsala observations find $S_{\mathrm{CO}}=129 \mathrm{Jy} \mathrm{km} \mathrm{s}^{-1}$, with an rms noise of $\pm 7 \mathrm{Jy} \mathrm{km} \mathrm{s}^{-1}$. Thus, within the uncertainties (rms noise, calibration), the BIMA map detects all the $\mathrm{CO}$ emission seen by Onsala at position $\mathrm{B}$, so the Onsala observations support the conclusion from the BIMA map that any $\mathrm{CO}$ emission at the eastern inner-disk arm is below the rms noise in either data set.

In the left panel of Figure $4, I_{\mathrm{CO}}$ contours are overlaid on the radio continuum $\lambda=20 \mathrm{~cm}$ image from the FIRST survey (Becker, White, \& Helfand 1995) in gray scale. The spatial resolution of the BIMA image (6". $6 \times 6$ ". 3 , HPBW) is a little lower than that of the radio image (5".4 HPBW). The radio continuum emission is strongly peaked on the starburst nucleus and symmetrically distributed about it.

The H I observations of Paper I are more sensitive to low column density gas than the $\mathrm{CO}$ observations presented here. We detected $\mathrm{H}$ I emission from the entire inner disk, 

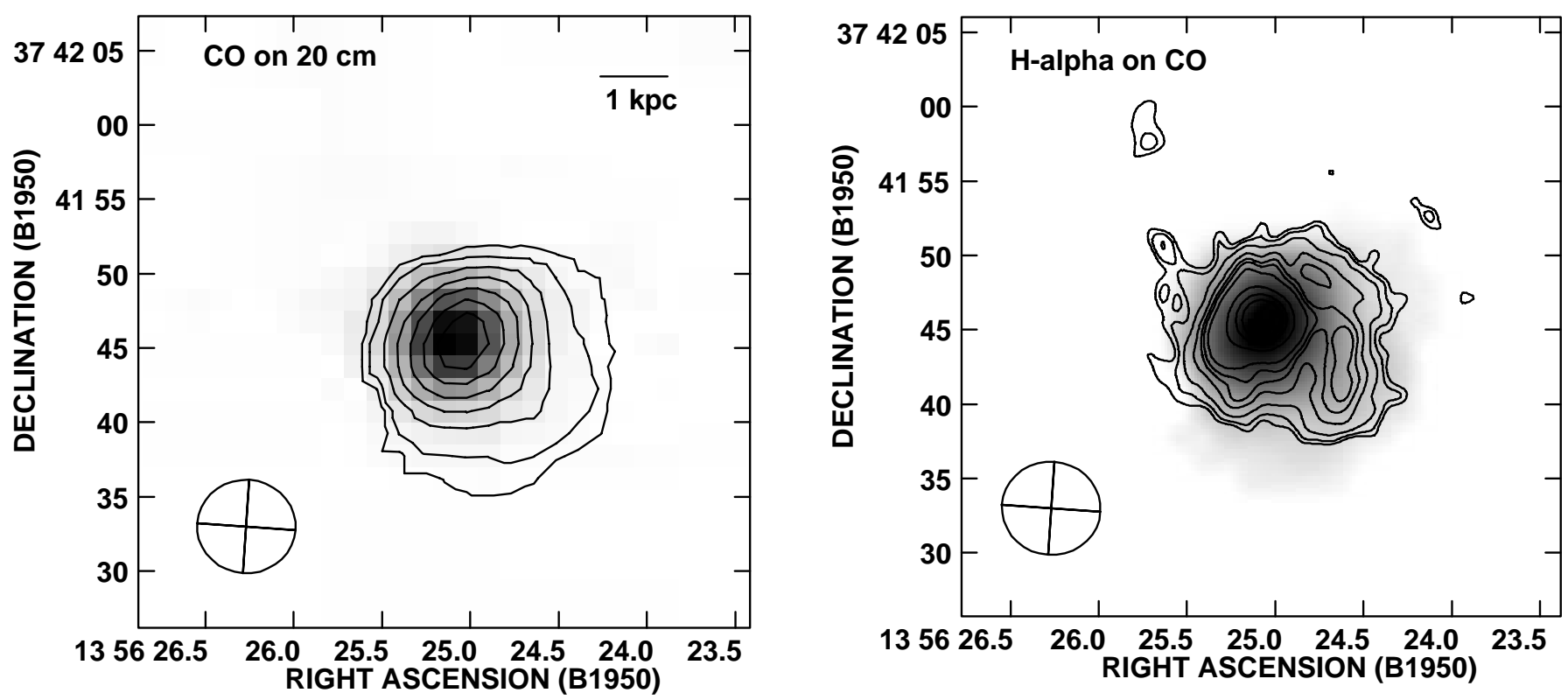

FIG. 4.-Left: Contours of BIMA ${ }^{12} \mathrm{CO} J=1 \rightarrow 0$ integrated intensity overlaid on the radio continuum $\lambda=20 \mathrm{~cm}$ image of the center of NGC 5394 from the FIRST survey in gray scale. Right: Contours of $\mathrm{H} \alpha$ emission overlaid on the BIMA CO integrated-intensity image of the center of NGC 5394 in gray scale. In the inner disk, $\mathrm{CO}$ and $\mathrm{H} \alpha$ emission have a similar lopsided spatial distribution with more emission from the southwestern side. In both panels, the beam symbol represents the $\mathrm{CO}$ resolution.

the southern tidal arm, and the inner third of the northern tidal arm (see Fig. 5). The line-of-sight H i column density $N(\mathrm{H} \mathrm{I})$ is greater on the western side than on the eastern side of the disk. The distributions of the ionized gas, atomic gas, and molecular gas in the disk of NGC 5394 all favor the

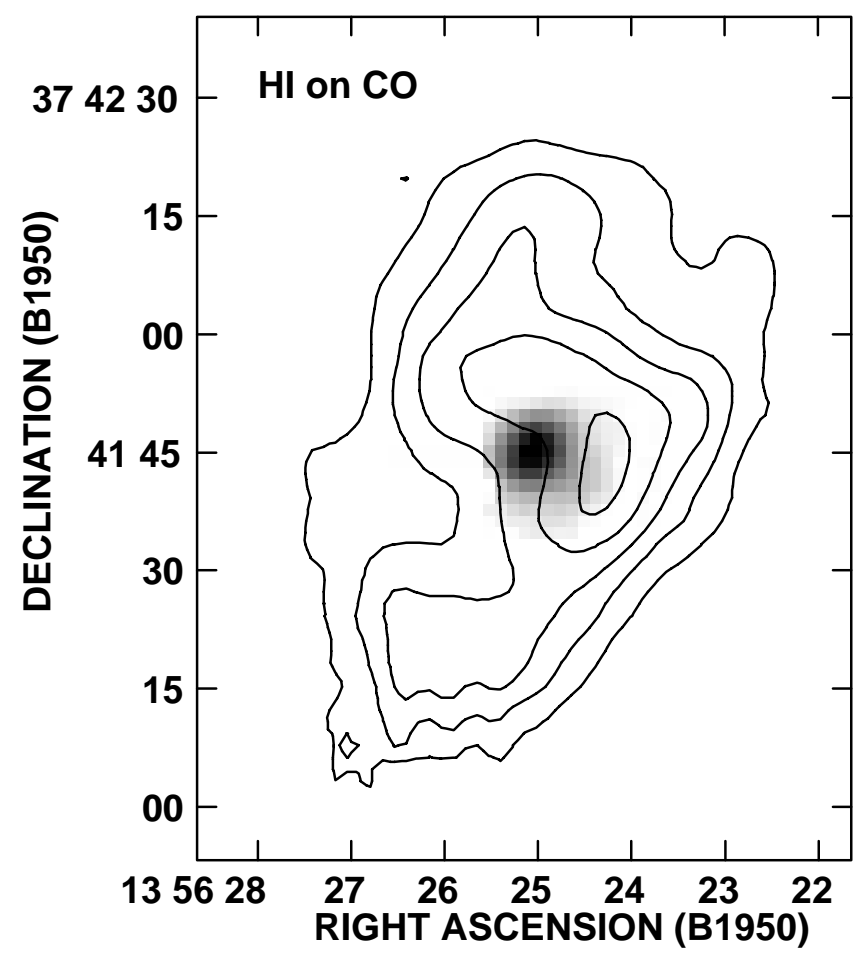

FIG. 5.-Contours of the $N(\mathrm{H} \mathrm{I})$ image (resolution $18^{\prime \prime}$ ) from Paper I overlaid on the BIMA $I_{\mathrm{CO}}$ image of NGC 5394 in gray scale. This displays the larger scale $\mathrm{H}$ I structure of the galaxy, whereas Figs. 3, 4, and 6 are enlargements of the central region. The $N(\mathrm{H} \mathrm{I})$ contour levels are at 2.0, 4.3, $4.8,6.1$, and $7.5 \times 10^{20}$ atoms $\mathrm{cm}^{-2}$. western or southwestern side. Unless driven by external forces, such a lopsided distribution of gas should not last more than a few shear times.

Roughly $80 \%$ of the BIMA CO emission from NGC 5394 is concentrated in the central $800 \mathrm{pc}$ (FWHM) starburst region, ${ }^{12}$ and the rest comes from the part of the inner disk south and west of the central starburst. After subtracting the $\mathrm{CO}$ emission from the $800 \mathrm{pc}$ starburst (assumed axisymmetric), we get the residual $I_{\mathrm{CO}}$ image displayed in the left panel of Figure 6 . The innermost western arm, $6^{\prime \prime}$ west of the nucleus, appears as an arm in $\mathrm{H} \alpha$ with the brightest $\mathrm{H} \alpha$ emission along its inside edge (see Fig. 6, right). Part of this arm is detected in $\mathrm{CO}$ emission, with a maximum $I_{\mathrm{CO}}$ of $31 \mathrm{~K} \mathrm{~km} \mathrm{~s}^{-1}$, equivalent to a line-of-sight column density $N\left(\mathrm{H}_{2}\right)$ of $7 \times 10^{21} \mathrm{H}_{2} \mathrm{~cm}^{-2}$. The $P-V$ diagrams in Figure 7 are for a slice along the declination axis, $6^{\prime \prime}$ west of the nucleus, and thus display the emission from the innermost western arm. These diagrams show that $\mathrm{CO}$ and $\mathrm{H} \alpha$ have a similar distribution of velocities, as well as similar mean velocities at this arm. Since the $\mathrm{H} \alpha$ resolution is 2".4, the $\mathrm{H} \alpha$ $P-V$ diagram in Figure 7 contains negligible emission from the starburst, whereas the $\mathrm{CO} P-V$ diagram contains some starburst emission.

Before correction for the velocity gradient across the beam, the $\mathrm{CO}$ one-dimensional velocity dispersion at the innermost western arm is $15 \mathrm{~km} \mathrm{~s}^{-1}$. To correct for the velocity gradient, we subtract the velocity difference across the Gaussian beam in quadrature. We have confirmed with numerical experiments that subtracting in quadrature is the appropriate correction (for plausible values of linear shear). This reduces the $\mathrm{CO}$ velocity dispersion to $14 \mathrm{~km} \mathrm{~s}^{-1}$. Since estimates of the cloud-cloud $\mathrm{CO}$ velocity dispersion in the Milky Way range from 3 to $9 \mathrm{~km} \mathrm{~s}^{-1}$, with a median value of

12 The $800 \mathrm{pc}$ size is from a Gaussian fit to the $\lambda=20 \mathrm{~cm}$ radio continuum and $\mathrm{H} \alpha$ emission, deconvolved from the point-spread function. 

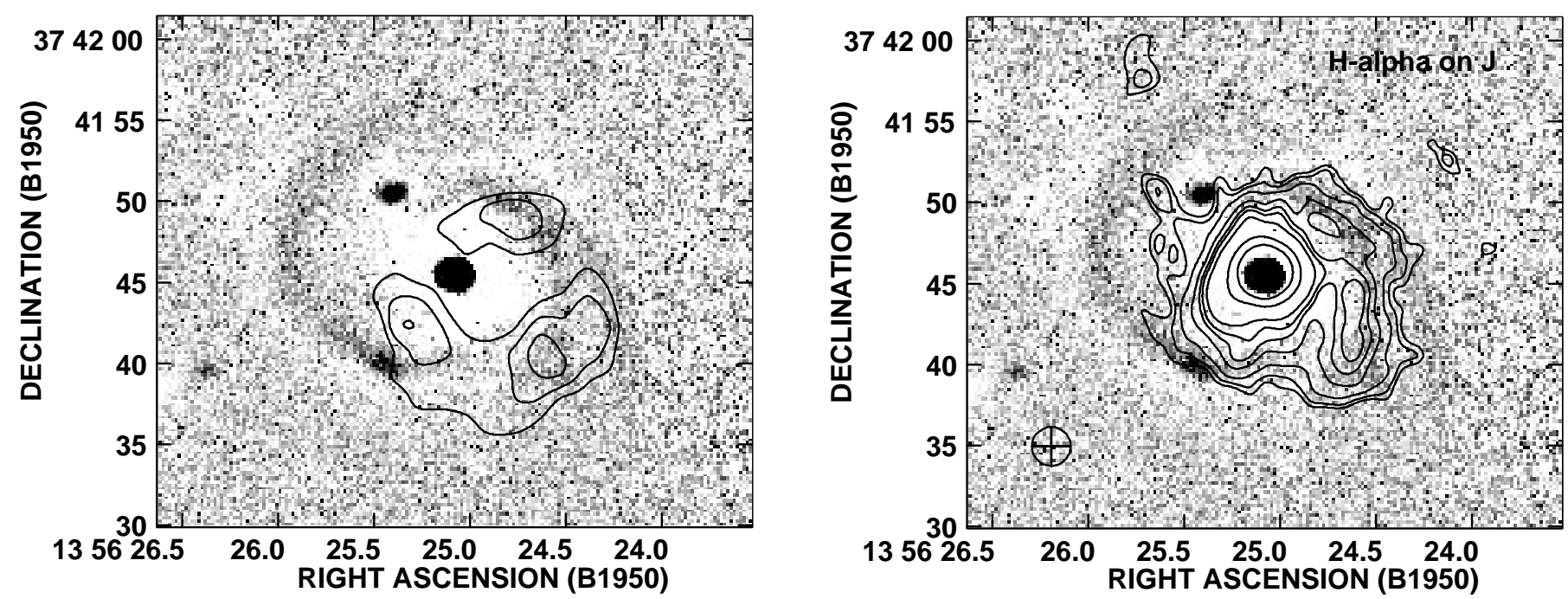

FIG. 6.-Left: Contours of the residual $I_{\mathrm{CO}}$ from NGC 5394 after subtracting the emission from the central starburst (assumed axisymmetric). These are overlaid on the unsharp-masked $J$-band image in gray scale to indicate the correspondence between the residual CO-emission and part of the innermost western arm. The $I_{\mathrm{CO}}$ contours are at $6,9,12 \mathrm{Jy}_{\text {beam }}^{-1} \mathrm{~km} \mathrm{~s}^{-1}$ and the rms noise is $2.8 \mathrm{Jy}$ beam $^{-1} \mathrm{~km} \mathrm{~s}^{-1}$. Right: Contours of H $\alpha$ emission overlaid on the unsharp-masked $J$-band image in gray scale. The beam symbol represents the $\mathrm{H} \alpha$ resolution.

$4 \mathrm{~km} \mathrm{~s}^{-1}$ (Combes 1991), the CO velocity dispersion at the innermost western arm seems to be somewhat higher than normal for galaxy disks. If attributed to unresolved in-disk streaming motions, such motions would need to be large because the disk is viewed nearly face-on (inclination $i=15^{\circ}$ ). Over much of the disk of NGC 5394, Paper I found $\mathrm{H}$ I velocity dispersions of $20-40 \mathrm{~km} \mathrm{~s}^{-1}$, which are high compared with the $\mathrm{H}$ i velocity dispersions of $6-13 \mathrm{~km} \mathrm{~s}^{-1}$ in undisturbed spirals (see references in Kaufman et al. 1997). Given the nearly face-on orientation of NGC 5394, these velocity dispersions probably represent mainly the $z$ component of the motions.
Paper I reported a weak ( 3 times rms noise) detection in the Onsala observations of $\mathrm{CO}$ emission from the southern tidal arm of NGC 5394, with $I_{\mathrm{CO}}=3.3 \mathrm{~K} \mathrm{~km} \mathrm{~s}^{-1}$. The BIMA observations detect no emission here. As the 2 times rms noise upper limit to $I_{\mathrm{CO}}$ in the BIMA data, we take $I_{\mathrm{CO}}=2 \times($ the $\mathrm{rms}$ noise per channel in the BIMA cube) $\times$ (the width of two channels) $/ \mathrm{PB}$, where $\mathrm{PB}$ is the primary-beam sensitivity of the mosaicked data. If the $\mathrm{CO}$ emission uniformly fills the Onsala $33^{\prime \prime}$ aperture on the southern tidal arm, it would be too faint to detect in the BIMA observations since the 2 times rms noise upper limit to $I_{\mathrm{CO}}$ at this position is $7.1 \mathrm{~K} \mathrm{~km} \mathrm{~s}^{-1}$ in the BIMA map.
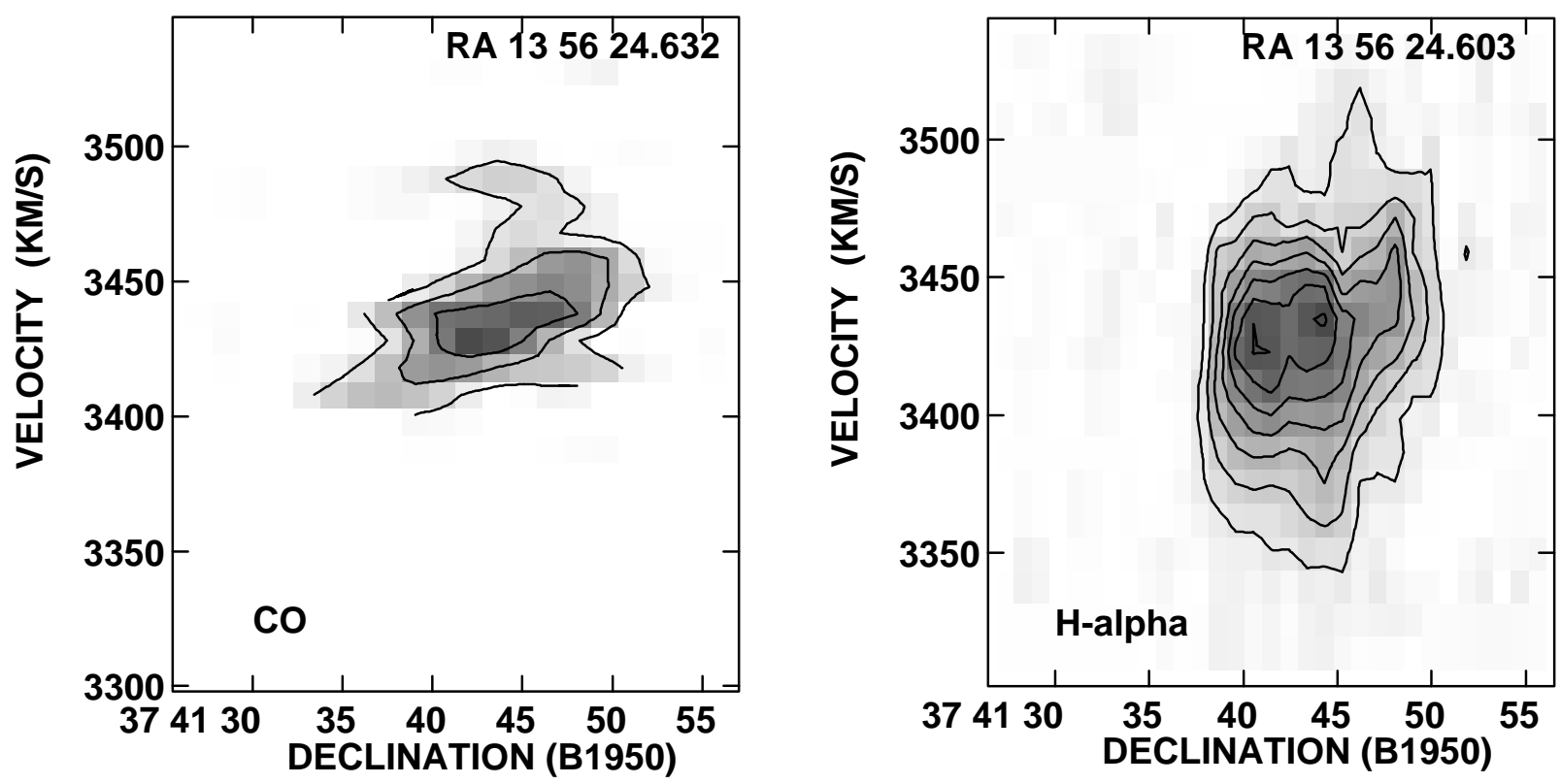

FIG. 7.-Position-velocity diagram in $\mathrm{CO}(l e f t)$ and $\mathrm{H} \alpha($ right $)$ of the emission from the innermost western arm of NGC 5394 . The cut is along the declination axis, $6^{\prime \prime}$ west of the nucleus. The contour levels in $\mathrm{CO}$ are at 2, 4, and 6 times the rms noise of $80 \mathrm{mJy}$ beam $^{-1}$. The contour levels in $\mathrm{H} \alpha$ are at $4,8,12,16$, 20,24 , and 28 times the rms noise. 


\section{THE EASTERN INNER-DISK ARM OF NGC 5394}

The eastern and the outer of the two western inner-disk arms are both devoid of ongoing star formation. No CO emission is detected from the eastern inner-disk arm. This is probably also the case for the outer of the two western inner-disk arms, but at our $\mathrm{CO}$ resolution, the situation there is contaminated by $\mathrm{CO}$ emission from the innermost western arm. Therefore we concentrate on the eastern innerdisk arm but assume that the same logic applies to the outer of the two western inner-disk arms.

Why is there no evidence of star formation in progress at the eastern inner-disk arm? We consider first the surface density of gas. $\mathrm{H}$ I emission from this arm is present with a

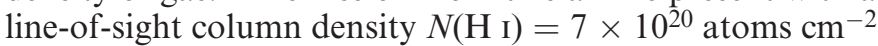
(measured with resolution $=11^{\prime \prime}=2.6 \mathrm{kpc} \mathrm{HPBW}$ in Paper I). The 2 times rms noise upper limit to $I_{\mathrm{CO}}$ at the eastern inner-disk arm in the BIMA data is $7.6 \mathrm{~K} \mathrm{~km} \mathrm{~s}^{-1}$. With the "standard" value of $X$, this corresponds to a 2 times rms noise upper limit to $N\left(\mathrm{H}_{2}\right)$ of $1.7 \times 10^{21} \mathrm{H}_{2} \mathrm{~cm}^{-2}$.

From observations of spiral galaxies, Kennicutt (1989) and Martin \& Kennicutt (2001) found that the instability parameter $Q_{\text {gas }} \leq 1.4$ for significant star formation to occur. The instability parameter $Q_{\text {gas }}=\kappa \sigma_{v \text {, gas }} / \pi G \mu_{\text {gas }}$, where $\kappa$ is the epicyclic frequency, $\sigma_{v}$,gas is the one-dimensional velocity dispersion in the gas, and $\mu_{\text {gas }}$ is the face-on surface density of gas. Paper I used $\mathrm{H}$ I data to determine values for these parameters. A position-velocity diagram in $\mathrm{H}$ I from Paper I (with $18^{\prime \prime}$ resolution) and a position-velocity diagram in $\mathrm{CO}$ (with 6".6 66 ".3 resolution) are displayed in Figure 8 and in the left panel of Figure 9, respectively, for a cut through the optical nucleus with abscissa along the projection line-ofnodes of the galaxy (position angle $=0^{\circ}$ ). The $\mathrm{H}$ i $P$ - $V$ diagram provides a rather messy "rotation curve" because NGC 5394 is viewed nearly face-on, the H I velocity disper- sion is high, there are streaming motions associated with the tidal arms and the bar, and the spatial resolution is low. From the H I data, Paper I concluded that the inner-disk arms are located on the solid-body part of the rotation curve and took the epicyclic frequency at galactocentric distance $R=10^{\prime \prime}$ (on the eastern inner-disk arm) as $\kappa=2 \Omega=37 \mathrm{~km}$ $\mathrm{s}^{-1} \mathrm{kpc}^{-1}$, where $\Omega$ is the orbital angular speed. ${ }^{13}$ Also, the inclination $i$ was estimated to be $9^{\circ}$ by comparing the observed value of $v_{\max } \sin i=27 \mathrm{~km} \mathrm{~s}^{-1}$ with the expected value of $v_{\max }=163 \pm 26 \mathrm{~km} \mathrm{~s}^{-1}$ from the $I$-band luminosity (see Paper I).

With the higher angular resolution of the CO $P$ - $V$ diagram we see that the rotation curve has a steeper rise in the inner disk than apparent from the $\mathrm{H}$ I data. It may have turned over by $R=10^{\prime \prime}$ in the south (see Fig. 9), but it is difficult to draw a firm conclusion because the emission at this radius is faint, the velocities are affected by the streaming along the bar, and the $\mathrm{CO}$ resolution is $\sim 6^{\prime \prime} .5$. At $R=10^{\prime \prime}$ we find $\Omega=41 \mathrm{~km} \mathrm{~s}^{-1} /(2.3 \mathrm{kpc} \sin i)$. The added information from the CO $P$ - $V$ diagram changes $v_{\max } \sin i$ to $41 \mathrm{~km}$ $\mathrm{s}^{-1}$ and thus changes our estimate of the inclination $i$ to $15^{\circ}$ and our estimate of the epicyclic frequency $\kappa$ to $[2(41 \mathrm{~km}$ $\left.\left.\mathrm{s}^{-1}\right)\right] /(2.3 \mathrm{kpc} \sin i)=1.4 \times 10^{2} \mathrm{~km} \mathrm{~s}^{-1} \mathrm{kpc}^{-1}$ at $10^{\prime \prime}$, if in solid-body rotation, or a factor of $\sqrt{2}$ less if on the flat part of the rotation curve.

Since the distribution of gas is lopsided, we use the surface density of gas on the eastern inner-disk arm at $R=10^{\prime \prime}$ to compute the instability parameter. For $\sigma_{v \text {,gas }}$, we adopt from Paper I the $\mathrm{H}$ I value of $30 \mathrm{~km} \mathrm{~s}^{-1}$ at the eastern inner-disk arm. The $\mathrm{H}$ I column density at this arm is $N(\mathrm{H} \mathrm{I})=7 \times 10^{20}$ atoms $\mathrm{cm}^{-2}$. Including a $36 \%$ by mass

${ }^{13}$ The value was misprinted in Paper I as $3.7 \mathrm{~km} \mathrm{~s}^{-1} \mathrm{kpc}^{-1}$.

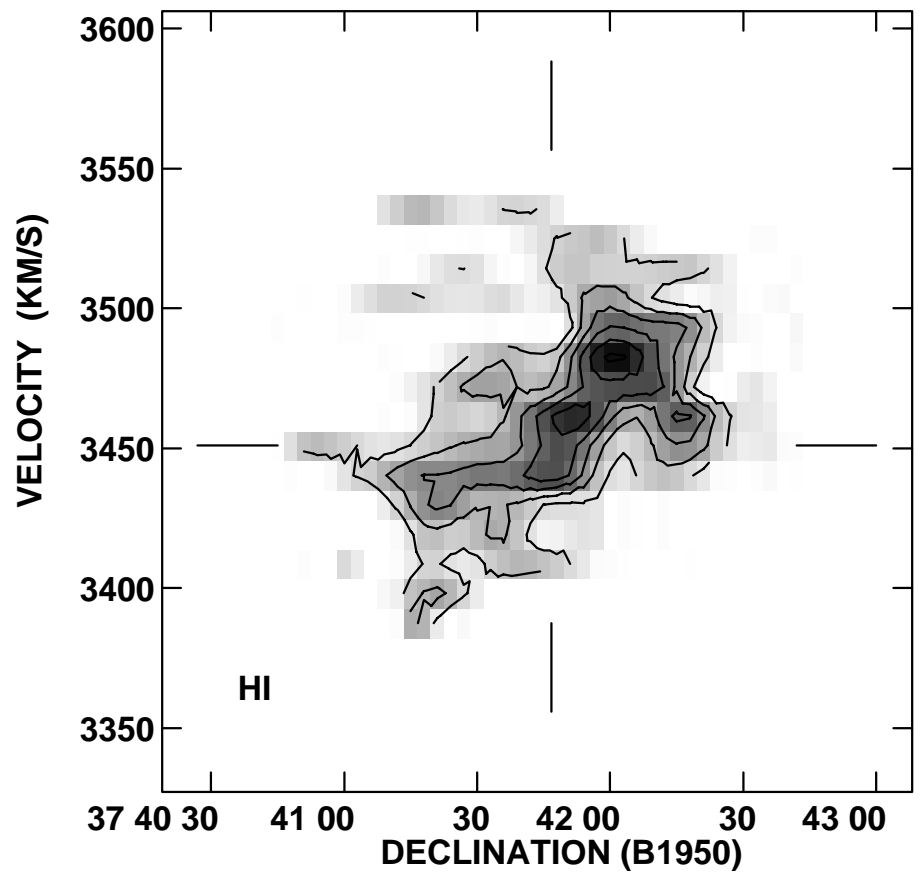

FIG. 8. - Position-velocity diagram in H I through the nucleus of NGC 5394, with the abscissa along the projection line of nodes of the galaxy (position angle $=0^{\circ}$ ). The large crosshairs mark the position and velocity of the optical nucleus. The $\mathrm{H}$ i contour levels are at 2, 3, 4, 5, 6, and 7 times the rms noise of $0.46 \mathrm{mJy}_{\text {beam }}{ }^{-1}$. The $\mathrm{H}$ I diagram includes emission over a significantly greater declination range but about the same velocity range as the $\mathrm{CO}$ emission in the $P$-V diagram in Fig. 9 (left $)$. 

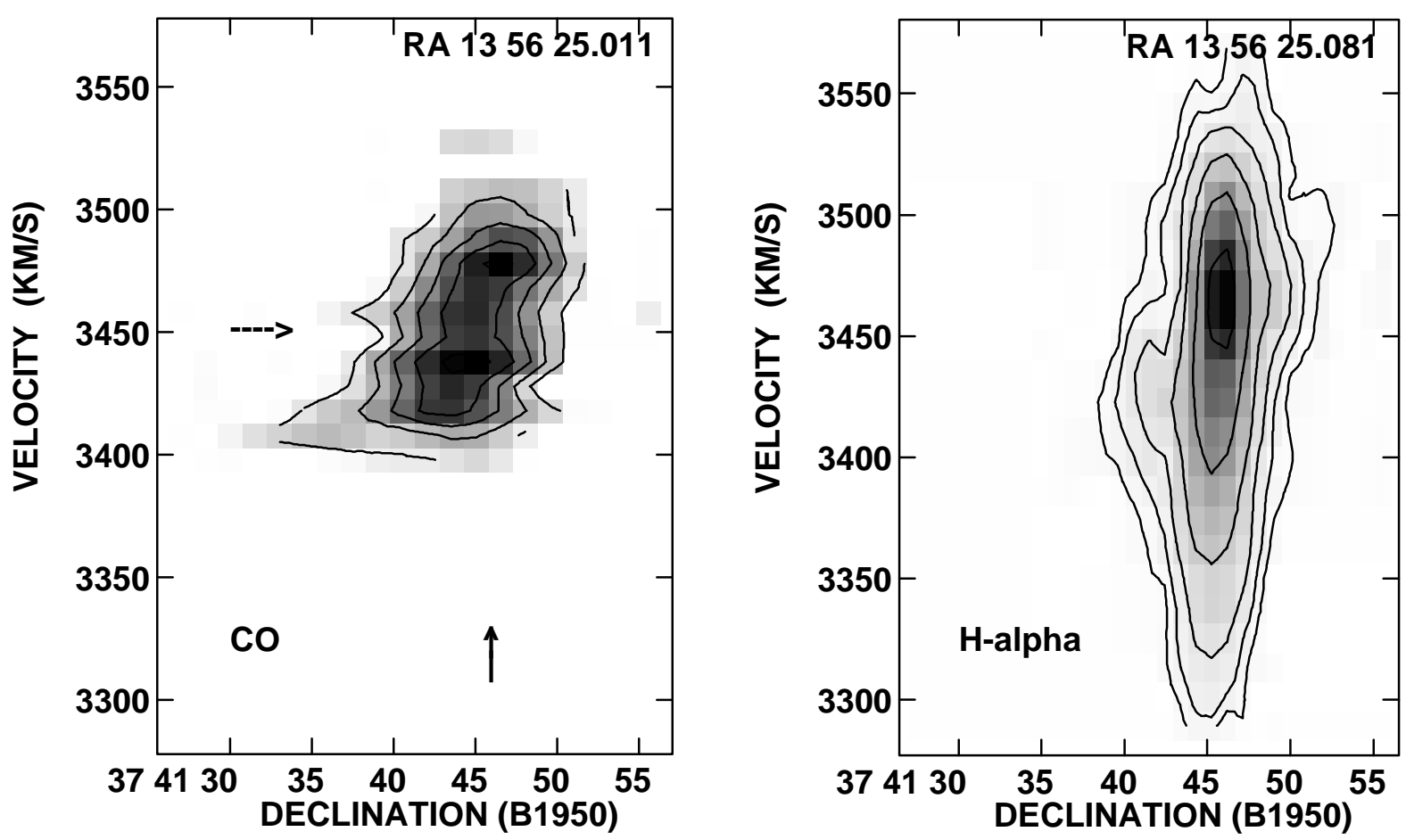

FIG. 9.-Position-velocity diagram in $\mathrm{CO}($ left $)$ and $\mathrm{H} \alpha$ (right) through the nucleus of NGC 5394, with the abscissa along the projection line of nodes of the galaxy (position angle $=0^{\circ}$ ). The contour levels in $\mathrm{CO}$ are at 2, 4, 6, 8, and 10 times the rms noise. The contour levels in $\mathrm{H} \alpha$ are at 4, 8, 16, 32, 64, and 128 times the rms noise. In the CO $P$ - $V$ diagram, arrows mark the declination of the nucleus and the long-slit $\mathrm{H} \alpha$ value for $v_{\text {sys. }}$ Unlike the $\mathrm{H} \alpha$ emission, the $\mathrm{CO}$ emission from the center of NGC 5394 is double-peaked in velocity, with one maximum at $3478 \mathrm{~km} \mathrm{~s}^{-1}$ and the other at $3438 \mathrm{~km} \mathrm{~s}^{-1}$. An extension to low velocities in $\mathrm{H} \alpha$ at the nucleus is not seen either in $\mathrm{CO}$ or in $\mathrm{H} \mathrm{I}$.

contribution from helium,

$$
\mu_{\mathrm{gas}}=(1+0.36)\left[N(\mathrm{H} \mathrm{I})+2 N\left(\mathrm{H}_{2}\right)\right] m_{p} \cos i,
$$

where $m_{p}$ is the proton mass. (The calculation of $\mu_{\mathrm{gas}}$ in Paper I ignored the contributions from helium and molecular gas.) Using the above 2 times rms noise upper limit to $N\left(\mathrm{H}_{2}\right)$ gives an upper limit to $\mu_{\text {gas }}$ of $43 M_{\odot} \mathrm{pc}^{-2}$ at $R=10^{\prime \prime}$ on the eastern inner-disk arm and a lower limit to $Q_{\text {gas }}$ of 5.0 if on the flat part of rotation curve. An upper limit to $Q_{\mathrm{gas}}$ is obtained by setting $N\left(\mathrm{H}_{2}\right)=0: Q_{\text {gas }}=29$ if on the flat part of the rotation curve. The values of $Q_{\text {gas }}$ should be increased by $\sqrt{2}$ if in solid-body rotation. Thus $Q_{\text {gas }}$ lies in the range 5 to 42 . Since this exceeds critical value of 1.4 from Kennicutt (1989) (see also Martin \& Kennicutt 2001), it appears that the instability parameter $Q_{\text {gas }}$ is presently too high for significant star formation at the eastern inner-disk arm.

For comparison, star formation is presently occurring on the innermost western arm and $\mathrm{CO}$ emission is detected from a portion of that arm. To compute $Q_{\text {gas }}$ there, we take our peak value of $I_{\mathrm{CO}}$ at this arm, which corresponds to an $N\left(\mathrm{H}_{2}\right)$ of $6.9 \times 10^{21} \mathrm{H}_{2} \mathrm{~cm}^{-2}$ and use our measured $\mathrm{CO}$ velocity dispersion of $14 \mathrm{~km} \mathrm{~s}^{-1}$ for $\sigma_{v, \text { gas }}$ since $N\left(\mathrm{H}_{2}\right)$ dominates $N(\mathrm{H} \mathrm{I})$ here. Then, with solid-body rotation, we get $Q_{\text {gas }} \sim 1$. So the Toomre gravitational instability criterion seems to explain the presence of star formation on the innermost western arm provided the value of the conversion factor $X$ is not appreciably less than the standard value.

The simple answer to the question of why there is no evidence of ongoing star formation at the eastern inner-disk arm or at the outer of the two western inner-disk arms is that there is presently too little molecular gas there. The question becomes why is there so little molecular gas associated with optically bright spiral arms.

\section{CENTRAL STARBURST IN NGC 5394}

\subsection{CO Flux, Star Formation Rate, and Extinction}

The total $S_{\mathrm{CO}}$ in NGC 5394 detected by BIMA is $117 \mathrm{Jy}$ $\mathrm{km} \mathrm{s}^{-1}$. Of this, approximately $97 \mathrm{Jy} \mathrm{km} \mathrm{s}^{-1}$ is from the starburst region (on the assumption of east-west symmetry for the starburst). Using the adopted standard value of $X$, we make the following comparison between the NGC 5394 starburst and the M82 starburst. The NGC 5394 starburst has a radio continuum luminosity at $\lambda=20 \mathrm{~cm}$ of $7.7 \times 10^{21} \mathrm{~W} \mathrm{~Hz}^{-1}$ (Paper I), a linear size of $800 \mathrm{pc}$, and an $\mathrm{H}_{2}$ mass $M\left(\mathrm{H}_{2}\right)$ of $1.9 \times 10^{9} M_{\odot}$ (excluding helium). The M82 starburst has a radio continuum luminosity of $1.0 \times 10^{22} \mathrm{~W} \mathrm{~Hz}^{-1}$, a size of $600 \mathrm{pc}$ (Condon et al. 1990), and an $M\left(\mathrm{H}_{2}\right)$ measured by Wild et al. (1992) for a region with diameter $800 \mathrm{pc}$ and scaled to the same value of $X$ of $4.9 \times 10^{8} M_{\odot}$. Thus, compared with the M82 starburst, the starburst in NGC 5394 has about the same linear size, 77\% of the $\lambda 20 \mathrm{~cm}$ radio continuum luminosity, but $\sim 4$ times the CO luminosity. It may be that the M82 starburst is in a more advanced state and has already used up more of the gas. Rieke et al. (1993) suggested that the M82 starburst consists of two starbursts, one of which started $3 \times 10^{7} \mathrm{yr}$ ago and the other, $5 \times 10^{6}$ yr ago. Vanzi, Alonso-Herrero, \& Rieke (1998) found that the starburst in NGC 5394 is several megayears past the peak star formation rate.

For the position of the nucleus, we take the location of the bright radio continuum peak in the FIRST image. (The 
stated uncertainty of the FIRST survey position is 0".4). There is negligible displacement between the surface brightness maxima in the radio continuum, $J$ band, and $\mathrm{H} \alpha$. The $I_{\mathrm{CO}}$ maximum is $0 . " 8$ west and $0 . " 4$ south of the radio continuum position. Since the positional uncertainty of the $\mathrm{CO}$ maximum is less than 0.6 , the offset between the $I_{\mathrm{CO}}$ and radio continuum maxima is marginal. It could result from some depletion of the $\mathrm{CO}$ flux at the radio continuum maximum, e.g., by photodissociation of $\mathrm{CO}$ or by star formation consuming the gas. Gaussian fits to the emission (Table 3) show that the $\mathrm{H} \alpha$ source and the radio continuum source in the center of NGC 5394 have the same size, $800 \mathrm{pc}$ (FWHM). The CO emission with a major axis of $1.1 \mathrm{kpc}$ (FWHM) is slightly more extended. The radio continuum emission from NGC 5394 is nonthermal with a spectral index of -0.7 , and $83 \%$ of the radio continuum flux density $S_{\nu}(20)$ of NGC 5394 at $\lambda=20 \mathrm{~cm}$ is from the starburst region (see Table 1 ).

There is no evidence that the emission from the center of NGC 5394 is dominated by an AGN. The optical and nearinfrared spectra (Leech et al. 1989; Vanzi, Alonso-Herrero, \& Rieke 1998) are H II region type. The radio continuum emission does not appear to be sufficiently compact to be mainly from an AGN. It is likely that most of the radio emission originates from supernova remnants or cosmic-ray electrons previously accelerated in supernovae, as is the case for the M82 starburst.

The measured $\mathrm{H} \alpha$ flux from the NGC 5394 starburst, not corrected for extinction, implies a radio continuum flux density from optically thin free-free emission $0.9 \%$ of the measured value of $S_{\nu}(20)$ (see Paper I). We can get a firm upper limit to the average visual extinction $A_{v}$ of the $\mathrm{H}$ II regions by assuming that all the measured $S_{\nu}(20)$ is free-free emission (clearly it is not, since the spectral index is nonthermal). The resulting upper limit, $A_{v}=1.28 \times A(\mathrm{H} \alpha)=6.5 \mathrm{mag}$, is unrealistically high since it assumes a thermal fraction at $\lambda 20 \mathrm{~cm}$ of $100 \%$. For a better estimate we choose the following two alternatives. In the M82 starburst, the thermal fraction at $\lambda 20 \mathrm{~cm}$ is $7 \%$, as determined by Golla, Allen, \& Kronberg (1996) from a comparison of [Ne II] $12.8 \mu \mathrm{m}$ and radio continuum emission. If the same thermal fraction applies to the NGC 5394 starburst, then the intrinsic $\mathrm{H} \alpha$ flux is a factor of 7.5 greater than the measured $\mathrm{H} \alpha$ flux, and the average extinction for star-forming regions in the $\mathrm{NGC}$ 5394 central starburst is $A_{v}=1.28(2.5) \log 7.5=2.8 \mathrm{mag}$. If instead we adopt the $\lambda 20 \mathrm{~cm}$ thermal fraction of $\sim 13 \%$ typical of normal disk galaxies (Condon 1992), then the intrinsic $\mathrm{H} \alpha$ flux is a factor of 14 times the measured $\mathrm{H} \alpha$ flux, and the average extinction for the $\mathrm{H}$ II regions is $A_{v}=3.7 \mathrm{mag}$.

TABLE 3

Radio, H $\alpha$, ANd CO Emission From Center of NGC 5394

\begin{tabular}{|c|c|c|}
\hline Wavelength & $\begin{array}{c}\text { Source Size }^{\mathrm{a}} \\
\text { (FWHM, P.A.) }\end{array}$ & $\begin{array}{c}\text { Resolution } \\
\text { (HPBW) }\end{array}$ \\
\hline$\lambda 20 \mathrm{~cm}$ radio continuum ....... & $3 \prime \prime 5 \times 3 \prime \prime 4,66^{\circ}$ & $5.4 \times 5 " .4$ \\
\hline $\mathrm{H} \alpha \ldots \ldots \ldots$ & $3 \prime .5 \times 3 " .1,118^{\circ}$ & $2.4 \times 2.4$ \\
\hline $\mathrm{CO} J=1 \rightarrow 0$ & $4 " .7 \times 3 . .8,148^{\circ}$ & $6 " 6 \times 6 . .3$ \\
\hline
\end{tabular}

a From Gaussian fit to emission, deconvolved from pointspread function.
Attributing all of the IRAS $60 \mu \mathrm{m}$ flux of the Arp 84 system to NGC 5394, Leech et al. (1989) derived a luminosity ratio $L(60 \mu \mathrm{m}) / L(\mathrm{H} \alpha)$ of 2000 for the NGC 5394 starburst. For the disks of bright spiral galaxies, Leech et al. (1989) found $L(60 \mu \mathrm{m}) / L(\mathrm{H} \alpha)$ equals 20-200 (based on data from Persson \& Helou (1987) in which the $\mathrm{H} \alpha$ flux of the disk was corrected for a mean extinction of $1.1 \mathrm{mag}$ at $\mathrm{H} \alpha$ ). With a resolution of $4.8 \times 1.5$ at $60 \mu \mathrm{m}$, the $I R A S 60 \mu \mathrm{m}$ flux of Arp 84 includes both galaxies. To determine what fraction of the IRAS flux is from the NGC 5394 starburst, we use the FIR-nonthermal radio correlation for spiral galaxies. Since Paper I finds that $29 \%$ of the radio continuum flux from the galaxy pair is from the central starburst in NGC 5394, we attribute $29 \%$ of the IRAS $60 \mu \mathrm{m}$ flux to the NGC 5394 starburst. This reduces the value of $L(60 \mu \mathrm{m}) / L(\mathrm{H} \alpha)$ for the starburst to $\sim 600$. If we adopt the range of values $(2.8-3.7$ mag) for the average $A_{v}$ from the thermal fraction argument, then the value of $L(60 \mu \mathrm{m}) / L(\mathrm{H} \alpha)$ is further reduced to the range $40-80$, similar to values found in the disks of bright spirals. Adoption of an average $A_{v}$ of 3-4 mag gives a plausible consistency between the IRAS $60 \mu \mathrm{m}$ flux, the radio continuum, and the $\mathrm{H} \alpha$ flux. It thus appears that the intrinsic $\mathrm{H} \alpha$ flux is an order of magnitude greater than the observed $\mathrm{H} \alpha$ flux. In that case the dereddened $\mathrm{H} \alpha$ luminosity of the starburst region is $\sim 8 \times 10^{41} \mathrm{ergs} \mathrm{s}^{-1}$. Then, taking the star formation rate $(\mathrm{SFR})=7.9 \times 10^{-42} L(\mathrm{H} \alpha) M_{\odot}$ $\mathrm{yr}^{-1}$ from Kennicutt (1998), we get an SFR of $6 \pm 2 M_{\odot}$ $\mathrm{yr}^{-1}$ for the starburst region.

The $\mathrm{H} \beta$ to $\mathrm{H} \alpha$ emission-line ratio (Balmer decrement) measured for the NGC 5394 nucleus by Leech et al. (1989) with a 1.5 wide slit gives a visual extinction $A_{v}$ of $1.3 \mathrm{mag}$ (assuming case $\mathrm{B}$ recombination). They find broadband optical colors, uncorrected for reddening, similar to those of $\mathrm{G}$ stars. They note that the $\mathrm{H} \alpha$ flux is roughly consistent with the number of ionizing stars implied by the broadband optical fluxes. In the BIMA data the maximum $I_{\mathrm{CO}}$ in the nucleus is $1.7 \times 10^{2} \mathrm{~K} \mathrm{~km} \mathrm{~s}^{-1}$, and the mean value of $I_{\mathrm{CO}}$ for the starburst is $66 \mathrm{~K} \mathrm{~km} \mathrm{~s}^{-1}$. With the standard value of the conversion factor $X$, the maximum $I_{\mathrm{CO}}$ implies a column density $N\left(\mathrm{H}_{2}\right)=3.8 \times 10^{22} \mathrm{H}_{2} \mathrm{~cm}^{-2}$, and the mean $I_{\mathrm{CO}}$, a column density $N\left(\mathrm{H}_{2}\right)=1.5 \times 10^{22} \mathrm{H}_{2} \mathrm{~cm}^{-2}$. For a standard dust-to-gas ratio with $A_{v} / N_{\text {gas }}=0.53 \times 10^{-21}$ mag (atoms $\left.\mathrm{cm}^{-2}\right)^{-1}$ (Bohlin, Savage, \& Drake 1978), the mean CO column density implies a highly opaque region with $A_{v}=11$ mag to the midplane. Although it is known that the dust-to-gas ratio is proportional to metallicity $Z$ (Sodroski et al. 1995), we lack a reliable determination of the metallicity of the NGC 5394 starburst. Leech et al. (1989) note that the measured value of the $[\mathrm{N} \mathrm{II}] / \mathrm{H} \alpha$ emission-line ratio for the starburst is compatible with solar metallicity, but they warn that this is not conclusive because the ionization parameter is unknown.

How can we reconcile these three very different values for $A_{v}$ ? The small value of $A_{v}(1.3 \mathrm{mag})$ from the Balmer decrement, as compared with the average extinction (3-4 mag) from the thermal fraction argument, means that the observed optical flux originates from regions of small optical depth (near the edges of the clouds) and we are not seeing the optical photons emitted by stars and $\mathrm{H}$ II regions deeply embedded in opaque clouds. Thus, optically, most of the starburst is hidden from view, probably in a patchy distribution of highly opaque clouds, as Leech et al. (1989) suggest.

The value of $A_{v}(11 \mathrm{mag})$ deduced from the mean $I_{\mathrm{CO}}$ with standard values for $X$ and the dust-to-gas ratio exceeds the 
average $A_{v}$ of 3-4 mag from the radio thermal fraction versus $\mathrm{H} \alpha$ and $L(60 \mu \mathrm{m}) / L(\mathrm{H} \alpha)$ arguments. The most likely explanation is that the conversion factor $X=N\left(\mathrm{H}_{2}\right) / I_{\mathrm{CO}}$ in the starburst region is a factor of 3-4 below the standard value. Similar reductions in the value of $X$ have been obtained for high excitation regions in the centers of other galaxies (Wall et al. 1993; Regan 2000). For the Milky Way, Sodroski et al. (1995) concluded that $X$ is a factor of 3-10 lower near the Galactic center than in the disk at $R=2-7$ kpc. In the M82 starburst, Smith et al. (1991) and Wild et al. (1992) found that the value of $X$ is a factor of 3 below the standard value. It appears that the different physical conditions in a starburst nucleus (Brouillet \& Schilke 1993; Aalto et al. 1995,1997$)$ result in a smaller value of $X$ than in the main disk of a spiral galaxy. Kaufman et al. (1999b) calculate the dependence of $X$ for a photodissociation region on far-ultraviolet flux, density, column density, and metallicity, and they apply their model to the M82 starburst as an example. They can fit the M82 observations with clouds that have higher gas density and higher thermal pressures than GMCs in the Milky Way.

Use of the standard value of $X$ overestimates the extinction in the starburst region of NGC 5394 and consequently overestimates the total molecular mass of NGC 5394. If the value of $X$ is down by a factor of 3-4 in the starburst region, but not reduced outside of the starburst, then the total mass in gas in the galaxy is $(3.6 \pm 0.4) \times 10^{9} M_{\odot}$.

\subsection{Velocity Structure}

Figure 9 displays position-velocity diagrams through the nucleus of NGC 5394 in $\mathrm{CO}$ and in $\mathrm{H} \alpha$ (from the FabryPerot data of Paper I) with the abscissa along the projection line of nodes of the galaxy (position angle $=0^{\circ}$ ). In the Fabry-Perot $\mathrm{H} \alpha$ data the mean velocity at the nucleus is 7 $\mathrm{km} \mathrm{s}^{-1}$ greater than the long-slit value of $3451 \pm 12 \mathrm{~km} \mathrm{~s}^{-1}$ obtained by Karachentsev (1980); this indicates the uncertainty in the calibration of the Fabry-Perot $\mathrm{H} \alpha$ velocities at the NGC 5394 nucleus. The channel width is $12 \mathrm{~km} \mathrm{~s}^{-1}$ in the $\mathrm{H} \alpha$ cube and $10 \mathrm{~km} \mathrm{~s}^{-1}$ in the $\mathrm{CO}$ cube. These $P-V$ diagrams reveal two significant differences between the $\mathrm{CO}$ and $\mathrm{H} \alpha$ velocity distributions in the starburst region: (1) the $\mathrm{CO}$ emission is double peaked in velocity, whereas the $\mathrm{H} \alpha$ emission has a single velocity peak, and (2) in $\mathrm{H} \alpha$ a clear extension to lower velocities is seen at the nucleus but is absent in $\mathrm{CO}$. The CO emission has one maximum at $3478 \mathrm{~km} \mathrm{~s}^{-1}$ and the other at $3438 \mathrm{~km} \mathrm{~s}^{-1}$; the average of these two velocity peaks, $3458 \mathrm{~km} \mathrm{~s}^{-1}$, is close to the $\mathrm{H} \alpha$ systemic velocity. The separation in velocity implies a spatial separation, e.g., one velocity peak from the approaching side and the other from the receding side. This could result from some depletion of $\mathrm{CO}$ at the nucleus by photodissociation or consumption by star formation, or the molecular gas could be in a nuclear ring or in "twin peaks" near an inner Lindblad resonance (ILR). It also means that the two $\mathrm{CO}$ peaks are not spatially coincident with the peak in the $\mathrm{H} \alpha$ emission. If the double peaks in CO are in the disk plane of NGC 5394, then each has a velocity difference with respect to the nucleus of $\left(v_{\mathrm{obs}}-v_{\mathrm{sys}}\right) / \sin i=20 \mathrm{~km} \mathrm{~s}^{-1} / \sin 15^{\circ}=77 \mathrm{~km} \mathrm{~s}^{-1}$.

We made $\mathrm{CO}$ position-velocity diagrams along various position angles. The maximum separation between the two CO peaks is $\sim 2^{\prime \prime}$ (less than 1/3 the synthesized beamwidth), with a smaller spatial separation along the bar major axis than along the bar minor axis. It is only the velocity structure that enables us to identify these as separate features.

Figure 10 compares the $\mathrm{CO}$ line profile at the nucleus with the $\mathrm{H} \alpha$ line profile from Paper I. From a Gaussian fit to the $\mathrm{CO}$ line profile at the nucleus, we find that the $\mathrm{CO}$ central velocity is $3453 \mathrm{~km} \mathrm{~s}^{-1}$ at the $I_{\mathrm{CO}}$ maximum and that the FWHM is $88 \pm 2 \mathrm{~km} \mathrm{~s}^{-1}$. Paper I suggested that the extended blue wing of the nuclear $\mathrm{H} \alpha$ profile (a velocity asymmetry of $\sim 100 \mathrm{~km} \mathrm{~s}^{-1}$ ) could represent an outflow of ionized gas from the starburst, perpendicular to the disk. From the above extinction estimate a counterflow on the far side would not be visible in $\mathrm{H} \alpha$. On the assumption that the $\mathrm{H} \alpha$ nuclear profile consists of a symmetric Gaussian com-
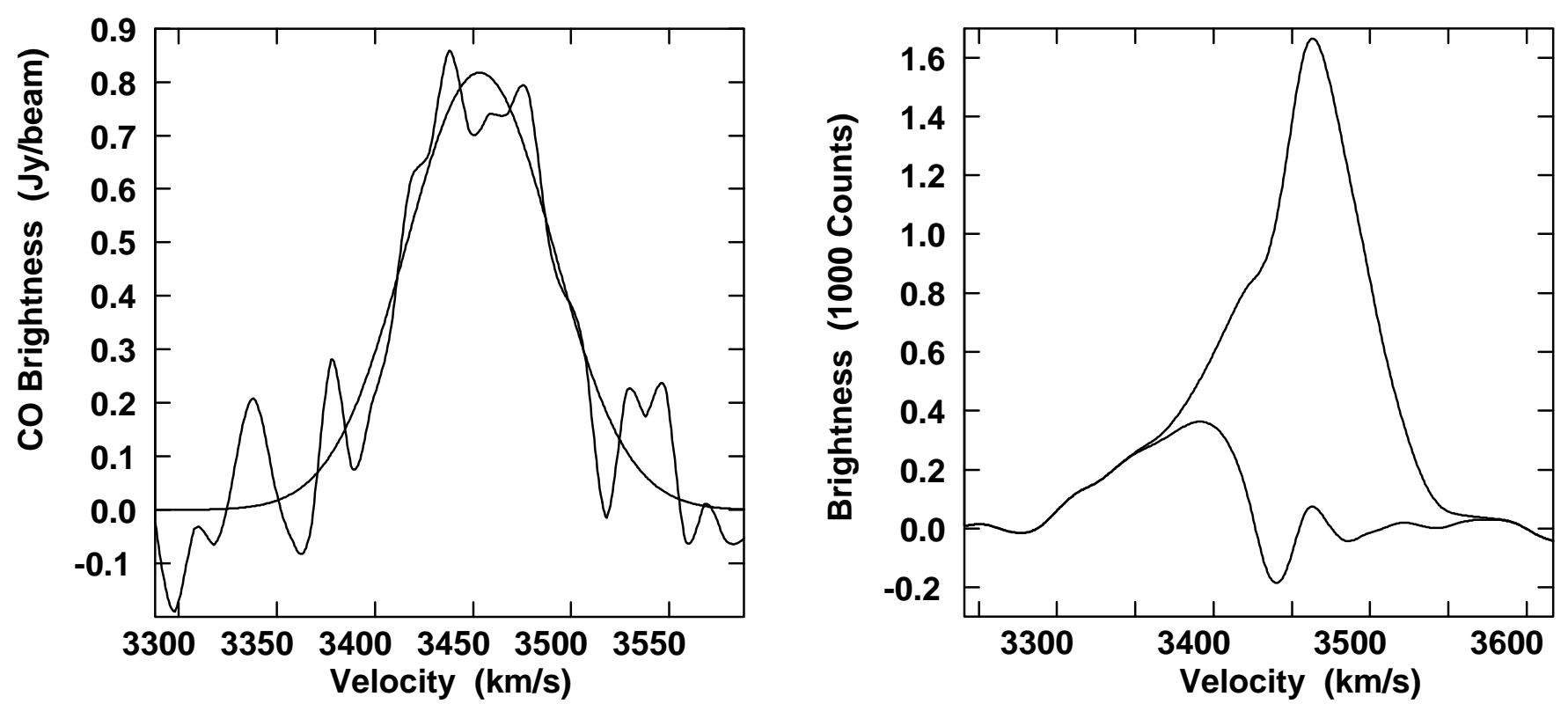

Fig. 10.- Line profiles in CO (left $)$ and $\mathrm{H} \alpha($ right $)$ at the nucleus of NGC 5394. The Gaussian fit to the CO line profile and the residual spectrum after fitting the $\mathrm{H} \alpha$ line profile (as described in the text) are also displayed. The dip at $3450 \mathrm{~km} \mathrm{~s}^{-1}$ in the CO profile occurs close to the long-slit $\mathrm{H} \alpha$ value of $3451 \mathrm{~km} \mathrm{~s}{ }^{-1}$ for $v_{\text {sys. }}$. 
ponent from the starburst in the nuclear disk plus an outflow/wind (visible on the near side only), we fitted the high velocity side of the profile with one side of a Gaussian with FWHM $=77 \mathrm{~km} \mathrm{~s}^{-1}$ and then subtracted the (full) Gaussian profile to obtain the residual $\mathrm{H} \alpha$ spectrum shown in Figure 10 for the outflow. In the $\mathrm{H} \alpha$ profile the maximum residual intensity in the blue wing (which we associate to an outflow) is $22 \%$ of the maximum in the original line profile. There is a hint of a feature (2-2.5 times the rms noise) in the CO profile at $80-100 \mathrm{~km} \mathrm{~s}^{-1}$ to the red of $v_{\text {sys }}$, but no clear evidence for a molecular counterpart to the $\mathrm{H} \alpha$ outflow component.

For comparison, in M82 the optical emission lines give an outflow speed of $\sim 600 \mathrm{~km} \mathrm{~s}^{-1}$ (Shopbell \& Bland-Hawthorn 1998), whereas the ${ }^{12} \mathrm{CO} J=1 \rightarrow 0$ and higher level $\mathrm{CO}$ molecular lines give an outflow speed of only $\sim 200 \mathrm{~km} \mathrm{~s}^{-1}$ (Shen \& Lo 1995; Nakai et al. 1987; Seaquist \& Clark 2001). Also, in M82, the line splitting that occurs in $\mathrm{H} \alpha$ is not seen in CO. If in the NGC 5394 starburst, the ratio of $\mathrm{H} \alpha$ to molecular outflow speeds is similar to that in M82, then in NGC 5394 it would be hard to identify an outflow in CO (that is, to distinguish between an outflow and a thick disk or spheroidal distribution) since the $\mathrm{H} \alpha$ outflow speed is only $\sim 100 / \cos 15^{\circ} \mathrm{km} \mathrm{s}^{-1}$.

The CO data have lower spatial resolution than the $\mathrm{H} \alpha$ data. If we correct for the gradient of the mean velocity field across the respective beams (by subtracting the velocity difference across the beam in quadrature), then the CO profile at the nucleus has a one-dimensional velocity dispersion of $31 \mathrm{~km} \mathrm{~s}^{-1}$, and the part of the $\mathrm{H} \alpha$ line profile that we attribute to the nuclear disk has a one-dimensional velocity dispersion of $30 \mathrm{~km} \mathrm{~s}^{-1}$. This probably represents mainly the $z$ component of the velocity dispersion. The molecular and $\mathrm{H} \alpha$ velocity dispersions at the NGC 5394 nucleus are about the same size as the $z$-component of the molecular velocity dispersion (30-54 $\mathrm{km} \mathrm{s}^{-1}$ ) in the Milky Way Galactic center estimated by Bally et al. (1988). The smaller value for the molecular velocity dispersion found in $\S 4$ pertains to the innermost western arm, not the nucleus.

\section{COMPARISON OF $\mathrm{CO}, \mathrm{H} \alpha$, AND H I VELOCITY FIELDS OF NGC 5394}

Figure 11 displays the ${ }^{12} \mathrm{CO} J=1 \rightarrow 0$ velocity field from the BIMA data, and Figure 12 the $\mathrm{H} \alpha$ velocity field (from Paper I). The $\mathrm{CO}$ velocity field covers only $\sim 2.5$ times the BIMA synthesized half-power beamwidth and does not reveal as much detail as the $\mathrm{H} \alpha$ velocity field. Given the difference in spatial resolution, the $\mathrm{CO}$ velocity field appears quite similar to the $\mathrm{H} \alpha$ velocity field. The similarity, despite $\mathrm{H} \alpha$ extinction, can be understood if the $\mathrm{H} \alpha$ - and CO-emitting layers in the disk have negligible shear perpendicular to the disk. The more extended VLA H I data in Paper I (18" resolution) provide the velocity field of the main disk and the tidal arms. In the main disk, the $\mathrm{H}$ i velocity field has kinematic minor axis at $90^{\circ} \pm 2^{\circ}$, so Paper I took the position angle of the galaxy's projection line of nodes as $0^{\circ} \pm 2^{\circ}$. The near side of the galaxy is the western side. In the CO and $\mathrm{H} \alpha$ velocity fields, the kinematic minor axis has rotated such that in the central $10^{\prime \prime}$ it has a very different position angle of $125^{\circ} \pm 5^{\circ}$ and is along the major axis of the $16^{\prime \prime}$ long primary bar. Some possible explanations for the rotation of a kinematic minor axis in the inner disk of a galaxy are streaming along a bar or axisymmetric radial motions. The sense of rotation of the kinematic minor axis of NGC 5394 is opposite to that expected for axisymmetric radial inflow in the disk. Axisymmetric radial expansion in the disk would rotate the kinematic minor axis in the sky plane

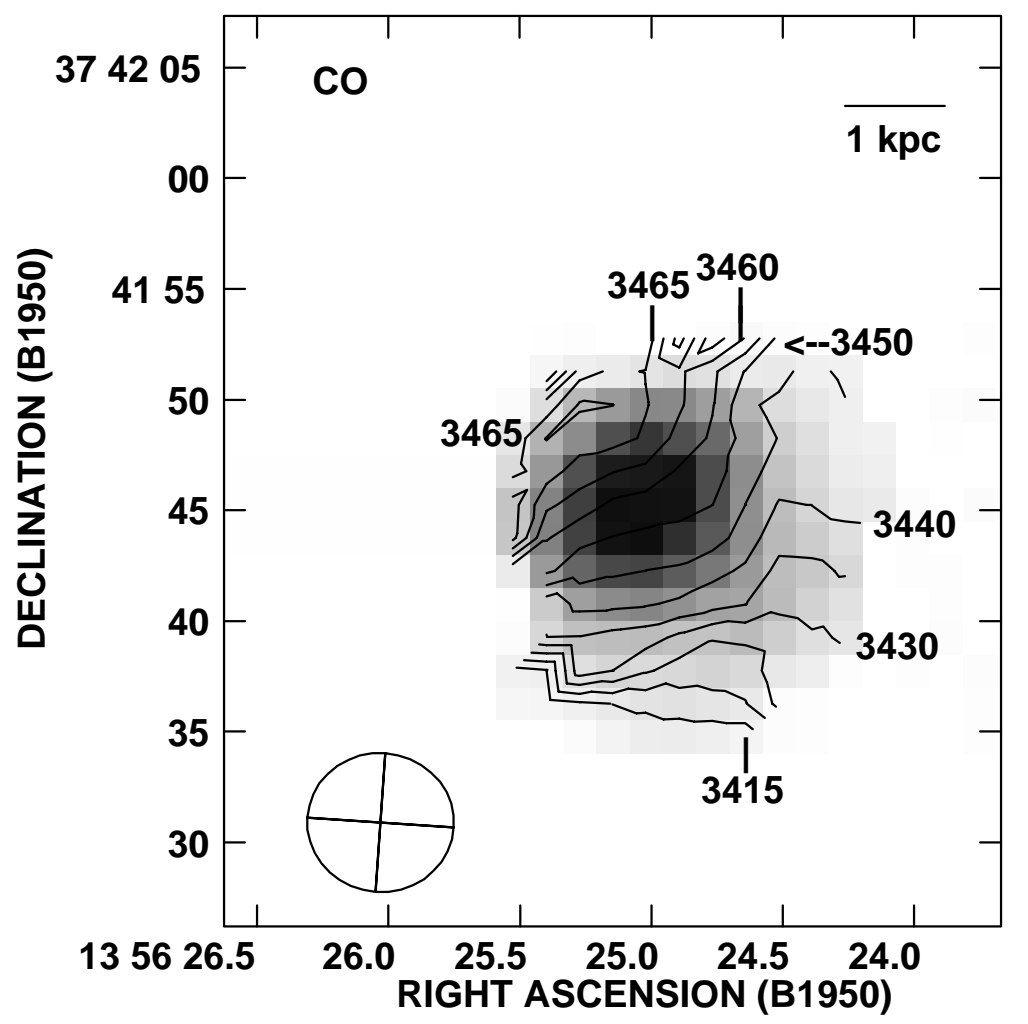

FIG. 11. - CO isovelocity contours overlaid on the CO integrated intensity image of NGC 5394 in gray scale 


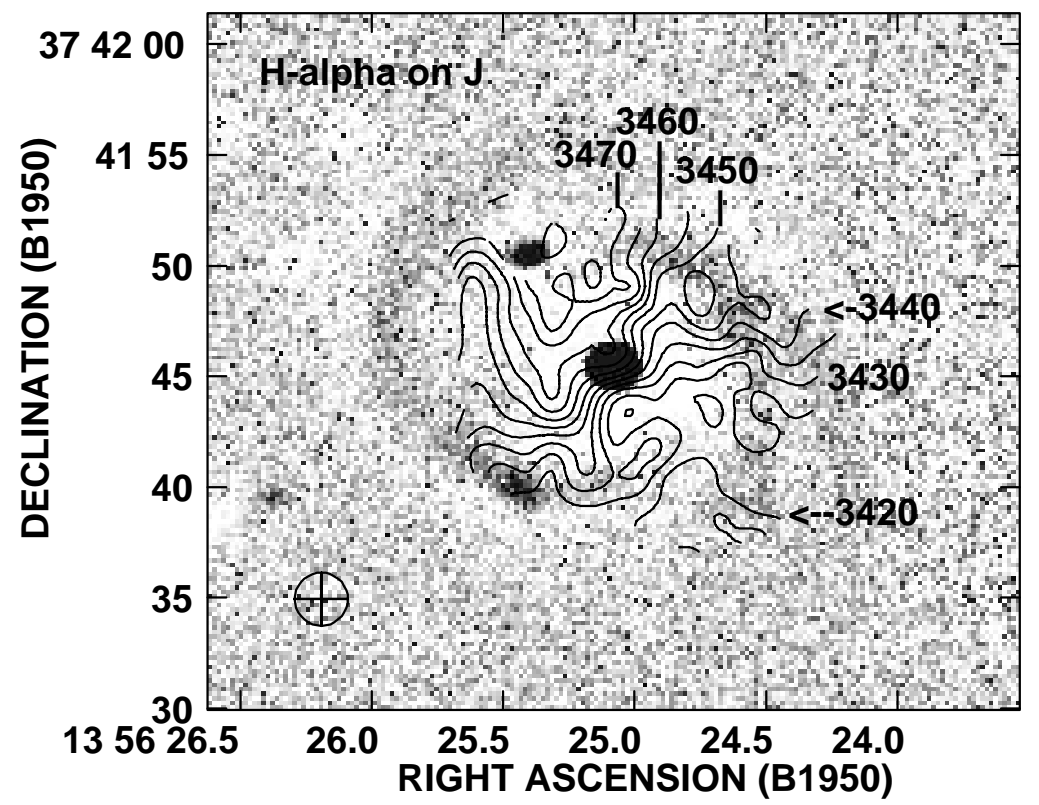

FIG. 12. $-\mathrm{H} \alpha$ isovelocity contours overlaid on unsharp-masked $J$-band image of NGC 5394 in gray scale. The beam symbol represents the H $\alpha$ resolution.

by $\tan \Delta \phi_{\text {minor }}=-\left(v_{\text {exp }} / v_{t}\right)$ sec $i$, where $\Delta \phi_{\text {minor }}$ equals the position angle of the minor axis of the projection minus the position angle of the kinematic minor axis of the central region, $v_{\exp }$ is the expansion speed (positive for expansion), and $v_{t}$ is the tangential speed (Wong \& Blitz 2000). Since the observed $\Delta \phi_{\text {minor }}=90^{\circ}-125^{\circ}=-35^{\circ}$ is negative, there is no evidence that axisymmetric inflow of gas into the inner disk is presently occurring. If axisymmetric expansion, the implied value of $v_{\text {exp }}$ is large, $0.68 v_{\mathrm{t}}$. The more likely interpretation of the rotation of the kinematic minor axis is that it represents gas moving in elliptical orbits around the bar. The inner-disk arms may have a different pattern speed than the primary bar, as they do not depart from the ends of the bar.

Since NGC 5394 is viewed nearly face-on, large in-plane velocity excursions would not appear very prominent in any line-of-sight velocity field. With its higher spatial resolution, the $\mathrm{H} \alpha$ velocity contours have more wiggles, indicative of streaming motions, than the $\mathrm{CO}$ velocity contours. Some of these appear associated with the innermost western arm. Additionally, it seems likely that the u-shaped kinks in the $\mathrm{H} \alpha$ velocity contours $3^{\prime \prime}$ southeast of the nucleus and the n-shaped velocity kinks $3^{\prime \prime}$ northwest of the nucleus are related to the possible secondary bar visible in the $J$ - and $K$-band images in Bushouse \& Stanford (1992). The bilateral symmetry of the velocity kinks at $3^{\prime \prime}$ provides kinematic evidence that this stellar feature is a coherent structure produced by a nonaxisymmetric gravitational potential. The position angle of the kinematic minor axis changes from $125^{\circ} \pm 5^{\circ}$ at $R \sim 5^{\prime \prime}$ to $102^{\circ} \pm 5^{\circ}$ at $R \sim 2^{\prime \prime}$; the kinks represent the transition between these two orientations. Thus the velocity field supports the interpretation that there are two nested bars, with the secondary bar trailing the primary bar by $30^{\circ}$. It is puzzling that the secondary bar is not seen in the higher resolution $J$-band image of Paper I.

\section{DISCUSSION OF RESULTS ON NGC 5394}

The BIMA ${ }^{12} \mathrm{CO} J=1 \rightarrow 0$ observations of the tidally distorted galaxy NGC 5394 reveal that: (1) about $80 \%$ of the $\mathrm{CO}$ emission detected by BIMA is concentrated in the 800 pc (FWHM) starburst region; (2) the extended $\mathrm{CO}$ and $\mathrm{H} \alpha$ emission in the inner disk have a similar lopsided spatial distribution, with an excess on the southwestern side of the disk, so star formation in the disk appears to be following the gas distribution; (3) no $\mathrm{CO}$ or $\mathrm{H} \alpha$ emission is detected from the bright, eastern inner-disk arm; (4) the estimated value of $X$ in the starburst is a factor of 3-4 below the standard value. The $\mathrm{H}$ I emission is also most prominent on the same (western) side of the disk as the $\mathrm{CO}$ and $\mathrm{H} \alpha$ emission, so in total gas NGC 5394 has a lopsided spatial distribution in the disk.

Reducing the value of the conversion factor $X$ by a factor of 3-4 in the starburst region, but using the standard value of $X$ outside of the starburst, we find from the Onsala $S_{\mathrm{CO}}$ that $M\left(\mathrm{H}_{2}\right) / M\left(\mathrm{H}_{\mathrm{I}}\right)=2.5-2.7$ and that $20 \%-25 \%$ of the atomic plus molecular gas in the galaxy is located in the central starburst. Galaxies with this high a value for $M\left(\mathrm{H}_{2}\right)$ / $M(\mathrm{H} \mathrm{I})$ are uncommon in the large sample of spiral galaxies surveyed by Casoli et al. (1998). Using the standard value for $X$, they find the mean value of $M\left(\mathrm{H}_{2}\right) / M\left(\mathrm{H}_{\mathrm{I}}\right)$ is 0.28 for Sa to Sc galaxies. Like other starburst galaxies, NGC 5394 is CO-rich. Also, using the standard value of $X$, Casoli et al. (1998) find that the average values of $\left[M\left(\mathrm{H}_{2}\right)+M(\mathrm{H} \mathrm{I})\right] /$ $D_{25}^{2}$ range from $5.6 \times 10^{6} M_{\odot} \mathrm{kpc}^{-2}$ for $\mathrm{Sa}$ galaxies to $1.0 \times 10^{7} M_{\odot} \mathrm{kpc}^{-2}$ for Sd galaxies, where $D_{25}$ is the isophotal diameter. They emphasize that $\left[M\left(\mathrm{H}_{2}\right)+M\left(\mathrm{H}_{\mathrm{I}}\right)\right] / D_{25}^{2}$ is simply a means of normalizing the gas mass to a measure of the galaxy size and should not be interpreted as a gas surface density. Since NGC 5394 is intrinsically oval, we set the value of $D_{25}$ for NGC 5394 equal to $2\left(R_{25}\right)_{\text {eff, where }}\left(R_{25}\right)_{\text {eff }}$ is defined in $\S 3$. Reducing the value of $X$ by a factor of 3-4 
in the starburst region but using the standard value of $X$ outside of the starburst gives $\left[M\left(\mathrm{H}_{2}\right)+M(\mathrm{H} \mathrm{I})\right] /$ $D_{25}^{2}=(1.2 \pm 0.1) \times 10^{7} M_{\odot} \mathrm{kpc}^{-2}$, which is typical of latetype spirals. The present lack of star formation in two of the three inner-disk arms results not from an overall paucity of gas in NGC 5394 but from its spatial distribution.

Paper I presented a galaxy encounter simulation that reproduces some of the main features of this galaxy pair with a collision that is prograde relative to NGC 5394 and retrograde at a high tilt angle relative to NGC 5395. In the model the response of NGC 5394 to the $\cos 2 \theta$ tidal potential of the encounter produces an eye-shaped (ocular) oval, which subsequently evolves into inner-disk arms, like those visible in NGC 5394. There is a west-versus-east time lag in the development of the ocular oval, with the eye-shaped rim forming first on what is now the eastern side of NGC 5394. The model keeps count of the number of gas particles in spherical volumes of radii 1.6 and $4 \mathrm{kpc}$ in the center of NGC 5394 as the encounter progresses. This is displayed in Figure 13. The limited particle resolution of the model prevents us from treating smaller volumes. The number of gas particles within $1.6 \mathrm{kpc}$ of the center builds up fairly steadily so that by the time of closest approach the mean gas density of this region is 3 times its initial value, and by the present time it has leveled off at 4 times its initial value. The model finds that the amount of material in the central regions is no longer increasing at the present time; recall that the observed velocity field shows no evidence of present axisymmetric inflow ( $\operatorname{see} \oint 7$ ).

The dominant tidal perturbation to NGC 5394 is an $m=2$ mode. This drives angular momentum transport, which produces the long tidal tails and inflow into the central region. The encounter also involves an $m=1$ term, which accounts for the time lag in the development of the

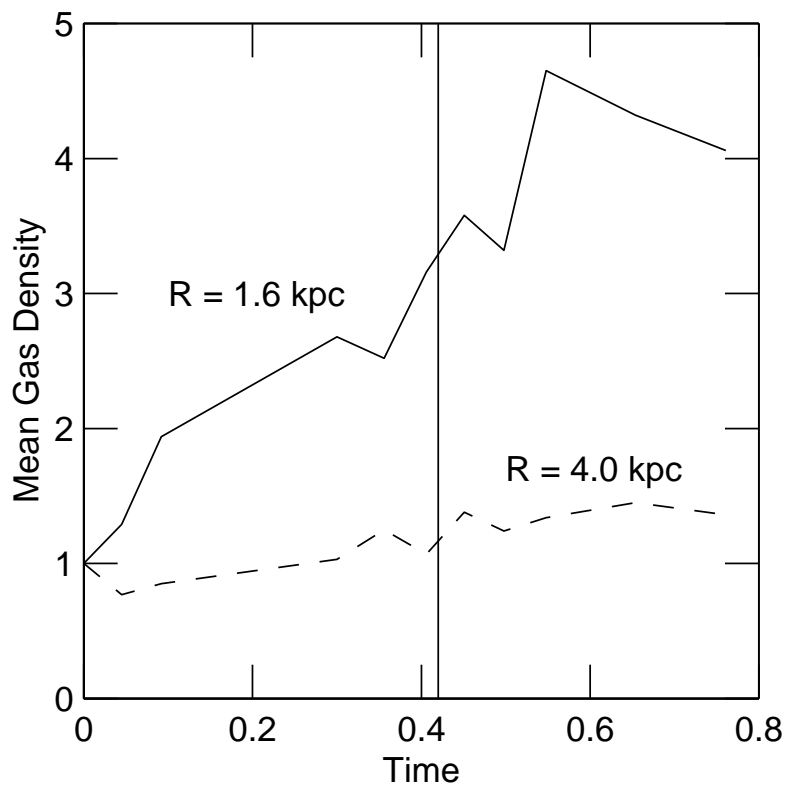

FIG. 13.-Model results on central densities of NGC 5394 as a function of time from the galaxy encounter simulation of Paper I. The curves represent the ratio of the mean density to the initial density for a spherical volume of radius $1.6 \mathrm{kpc}$ (solid curve) and for a spherical volume of radius 4.0 $\mathrm{kpc}$ (dashed curve). The vertical line marks the closest approach time, while the present time is near the latest time shown. The time unit is $4.2 \times 10^{7} \mathrm{yr}$. eastern and western sides of the ocular oval. The model for this system (described in Paper I) uses rigid halos (softened point-mass potentials). As the galaxies pass each other in the model, their disks do not quite interpenetrate, but their halos do. This results in a transient $m=0$ compression in the perturbation: before closest approach NGC 5394 compresses as a result of the increasing tidal force of the NGC 5395 halo. This contributes to the infall into the central region before closest approach, while after closest approach the inflow into the central region results from ocular-induced angular momentum transport and inertia.

The observed lopsided distribution of gas could result from an asymmetry in the collisional perturbation plus the loss of angular momentum by the gas in shocks. The latter is necessary to differentiate between the gas and the stars because the distribution of old stars does not show the same lopsidedness as the gas. In the model the asymmetry of the perturbation is evident as a time lag in the development of the two sides of the ocular oval. Since the stars form a collisionless system, they may respond differently than the gas, which can lose angular momentum in shocks. We offer the following speculative scenario for the inner-disk arms. The west-versus-east time lag in the development of the ocular oval may have led to a west-versus-east time lag in the formation of the inner-disk arms and to a west-versus-east time lag in the inflow of gas to the center. Gas at the eastern and the outer of the two western inner-disk arms underwent shocks (e.g., as a result of the bar, which may differ in pattern speed from the inner-disk arms) and flowed toward the center, leaving the stellar arms behind. This needs to be confirmed with more detailed simulations. Another possibility is that there may have been more gas inflowing toward the center on the western side.

Not all interacting galaxies are CO-rich (Horellou \& Booth 1997). From a comparison of the observed morphologies with models for prograde, in-plane, grazing encounters, Paper I concluded that the galaxies IC 2163, NGC 2535, and NGC 5394 form three distinct stages in the development of structures in this type of collision, with IC 2163 the least evolved and NGC 5394 the most evolved interaction. The "age" sequence is also seen in the star formation rates. IC 2163 has star formation typical of normal spiral disks (Elmegreen et al. 2001), and widespread CO emission is found in SEST observations with 44" resolution (Thomasson et al. 2002). In NGC 2535, star formation is somewhat enhanced (Bernlöhr 1993), the brightest $\mathrm{H} \alpha$ emission (Amram et al. 1989) occurs at the eastern and western ends of the eye-shaped oval at $R \approx 5 \mathrm{kpc}$ from the nucleus, and the Onsala observations with $33^{\prime \prime}$ resolution had clear detections of $\mathrm{CO}$ emission only from the central pointing (which includes the western apex) and from the portion of the tidal tail just beyond the eastern apex (Kaufman et al. 1997). Both positions have about the same integrated intensity $I_{\mathrm{CO}}$. With the standard value of $X$, NGC 2535 has $M\left(\mathrm{H}_{2}\right)$ / $M(\mathrm{H} \mathrm{I})=0.1$. In NGC 5394 most of the $\mathrm{CO}$ emission is from the central $800 \mathrm{pc}$ starburst region, and the ratio of molecular-to-atomic gas for the galaxy is typical of a starburst. Thus in prograde grazing encounters, there appears to be a time delay before conditions become suitable for a starburst. The length of the time delay depends on how rapidly post-encounter evolution proceeds. This small sample of three prograde encounters suggests that a central starburst may not develop until near the end of the ocular phase. 
It appears that the encounter caused a considerable amount of gas to lose angular momentum and fall into the center of NGC 5394, produced the lopsided distribution of gas in its inner disk, and may have increased the $M\left(\mathrm{H}_{2}\right) / M\left(\mathrm{H}_{\mathrm{I}}\right)$ ratio for the galaxy as a result of the conversion of atomic to molecular gas in the high density central region.

\section{NGC 5395 RESULTS}

We expected to find a compressed molecular ridge or clumped molecular gas at the following locations in NGC 5395: (1) in the dominant spiral arm on the eastern side of NGC 5395, since this coincides with a prominent radio continuum ridge (see Paper I); (2) in a stellar plus ionized gas "shell-like" structure (20" in diameter, marked on the northeast side of NGC 5395 in Fig. 1), which Paper I interpreted as a caustic produced by temporary convergence of orbits; (3) in the prominent dust lanes along the dominant spiral arm on the western side of NGC 5395. On the eastern side of the galaxy the dominant spiral arm is brighter in radio continuum and $\mathrm{H} \alpha$ emission, whereas on the western side of the galaxy it is brighter in $\mathrm{H} \mathrm{I}$. The face-on surface density of $\mathrm{H}$ I plus helium (measured with $11^{\prime \prime}$ resolution in Paper I) is $16-20 M_{\odot} \mathrm{pc}^{-2}$ at Onsala position $\mathrm{H}$ (at the main dust lane on the western side of NGC 5395) and $10 M_{\odot} \mathrm{pc}^{-2}$ at the caustic. NGC 5395 has an inclination of $65^{\circ}$.

We compare the ${ }^{12} \mathrm{CO} J=1 \rightarrow 0$ observations of NGC 5395 by the NRAO $12 \mathrm{~m}$ telescope (beam $=55^{\prime \prime}$ HPBW) (Zhu et al. 1999) and by Onsala (beam $=33$ HPBW) (Paper I) with the BIMA data presented here. None of these data sets has complete coverage of the galaxy. With three pointings at a spacing of $60^{\prime \prime}$, Zhu et al. (1999) measured a total integrated flux $S_{\mathrm{CO}}$ of $325 \pm 26 \mathrm{Jy} \mathrm{km} \mathrm{s}^{-1}$, equivalent to $M\left(\mathrm{H}_{2}\right)=7.1 \times 10^{9} M_{\odot}$ with a standard value of $X$. They then multiplied this flux by a factor of 1.7 to include $\mathrm{CO}$ emission outside of the region covered. Since $M(\mathrm{H}$ I $)$ is $1.4 \times 10^{10} M_{\odot}$ (Paper I), applying this factor gives an $M\left(\mathrm{H}_{2}\right) / M\left(\mathrm{H}_{\mathrm{I}}\right)$ ratio of 0.86 , so NGC 5395, which does not have a central starburst, is somewhat CO-rich compared with the typical spirals in the survey by Casoli et al. (1998) but not as CO-rich as its companion.

All three NRAO $12 \mathrm{~m}$ profiles have very broad CO line widths (Zhu et al. 1999). Correcting these for the $\mathrm{H}$ I velocity gradient across the $\mathrm{CO}$ beam and assuming that the $\mathrm{CO}$ emission fills the NRAO beamwidth parallel to the kinematic major axis (position angle $=173^{\circ}$ ) results in a onedimensional $\mathrm{CO}$ velocity dispersion of $\sim 80 \mathrm{~km} \mathrm{~s}^{-1}$. This is similar to the $\mathrm{H}$ I velocity dispersions of $50-70 \mathrm{~km} \mathrm{~s}^{-1}$ found in Paper I. As in the case of the $\mathrm{H}$ I line widths, the large value for the velocity dispersion probably results mainly because the line-of-sight intercepts gas at various radial distances in the disk and at various altitudes above the plane. Paper I presented evidence for three-dimensional disturbed structures in the $\mathrm{H}$ I gas. The broad $\mathrm{CO}$ line widths suggest that molecular gas may also participate in these threedimensional structures.

The Onsala and BIMA observations of NGC 5395 were not sufficiently sensitive to detect the molecular gas with high velocity dispersion seen by the NRAO $12 \mathrm{~m}$. The Onsala observations detected a CO component with low velocity dispersion in the northern part of the ring/pseudoring and measured an $S_{\mathrm{CO}}$ of $126 \mathrm{Jy} \mathrm{km} \mathrm{s}{ }^{-1}$ from the two pointings (labelled " $\mathrm{H}$ " and " $\mathrm{I}$ " in Fig. 1) centered 40" north of the nucleus. At positions $\mathrm{H}$ and I, the Onsala line profiles have FWHM line widths of 77 and $69 \mathrm{~km} \mathrm{~s}^{-1}$, respectively, and central velocities of 3314 and $3328 \mathrm{~km} \mathrm{~s}^{-1}$ (close to the $\mathrm{H}$ I mean velocities). From the $\mathrm{H}$ i velocity field (Paper I), we find a velocity gradient of $3.8 \mathrm{~km} \mathrm{~s}^{-1} \operatorname{arcsec}^{-1}$ at position $\mathrm{H}$ and $2.8 \mathrm{~km} \mathrm{~s}^{-1} \operatorname{arcsec}^{-1}$ at position I. If the $\mathrm{CO}$ emission fills the Onsala beam uniformly along the kinematic major axis, then the velocity gradient would produce a velocity difference across the beam of $125 \mathrm{~km} \mathrm{~s}^{-1}$ at position $\mathrm{H}$ and $90 \mathrm{~km} \mathrm{~s}^{-1}$ at position I. Since these values are appreciably greater than the FWHM line widths observed by Onsala, the $\mathrm{CO}$ emission does not fill the Onsala beam uniformly along the gradient of the velocity field (approximately north-south). The NRAO $12 \mathrm{~m}$ position $60^{\prime \prime}$ north of the nucleus includes part of Onsala positions $\mathrm{H}$ and I and the caustic feature. Its line profile consists of a narrow component with $v=3300-3360 \mathrm{~km} \mathrm{~s}^{-1}$ (the same velocity range as the emission found by Onsala at positions $\mathrm{H}$ and I) sitting atop the fainter, broad component with FWHM $=230 \mathrm{~km}$ $\mathrm{s}^{-1}$ (Zhu et al. 1999).

The BIMA observations detect very little $\mathrm{CO}$ emission from NGC 5395. In Figure 14 contours from BIMA channel maps for the appropriate velocity range are overlaid on the Digitized Sky Survey image in gray scale for a field containing the stellar caustic feature and positions $\mathrm{H}$ and I. This displays the best detection of $\mathrm{CO}$ emission in the BIMA observations of NGC 5395: a feature at the intersection of positions $\mathrm{H}$ and $\mathrm{I}$ with maximum $I_{\mathrm{CO}}=13 \mathrm{~K} \mathrm{~km} \mathrm{~s}^{-1}=3.7$ times the rms noise and $S_{\mathrm{CO}}=6 \mathrm{Jy} \mathrm{km} \mathrm{s}{ }^{-1}$, which is $5 \%$ of the $S_{\mathrm{CO}}$ measured by Onsala from the two pointings $\mathrm{H}$ and I. Its location mainly interior to the arc formed by the stellar caustic is surprising, as we had expected to find the $\mathrm{CO}$ emission in this field along the stellar caustic. The only other possible $\mathrm{CO}$ feature in Figure 14 is, at best, a marginal detection on the arm just south of the stellar caustic. The two channel at 2 times rms noise upper limit in this field corresponds to a face-on surface density, including helium, of $15 M_{\odot} \mathrm{pc}^{-2}$ for a standard value of $X$.

It is puzzling that BIMA missed almost all of the narrow velocity component to the $\mathrm{CO}$ emission at Onsala positions $\mathrm{H}$ and I. If the emission measured by Onsala were clumped in a linear feature $33^{\prime \prime} \times 6$ ". 5 , BIMA should have detected position $\mathrm{H}$ at 8.9 times the rms noise and position I at 4.7 times the rms noise (averaging the BIMA sensitivity over the Onsala aperture). The constraint imposed by the Onsala line width implies that the $\mathrm{CO}$ emission detected by Onsala has a north-south extent $\leq 20^{\prime \prime}$ at position $\mathrm{H}$ and $\leq 25^{\prime \prime}$ at position I. If the $\mathrm{CO}$ feature has dimensions $20^{\prime \prime} \times 33^{\prime \prime}$, then it could be below 2 times the rms noise at position I in the BIMA observations, but it should have been present at 2.9 times the rms noise at position $\mathrm{H}$ in the BIMA data. It may be that across the northern end of the $\mathrm{H}$ I, $\mathrm{H} \alpha$, radio continuum ring/pseudoring of NGC 5395, there is a $20^{\prime \prime}$-wide band of $\mathrm{CO}$ emission, possibly consisting of small, unresolved, molecular clouds, and that the east-west extent of the $\mathrm{CO}$ emission exceeds the largest scale visible to BIMA in C configuration.

Since the BIMA observations detected very little CO emission from NGC 5395, the molecular gas does not appear to be strongly concentrated in compressed ridges where we expected to find strong shock fronts. We are left with the puzzle that the eastern half of the ring/pseudoring is brighter in radio continuum and $\mathrm{H} \alpha$ emission, whereas the western half has a higher column density of total gas. 


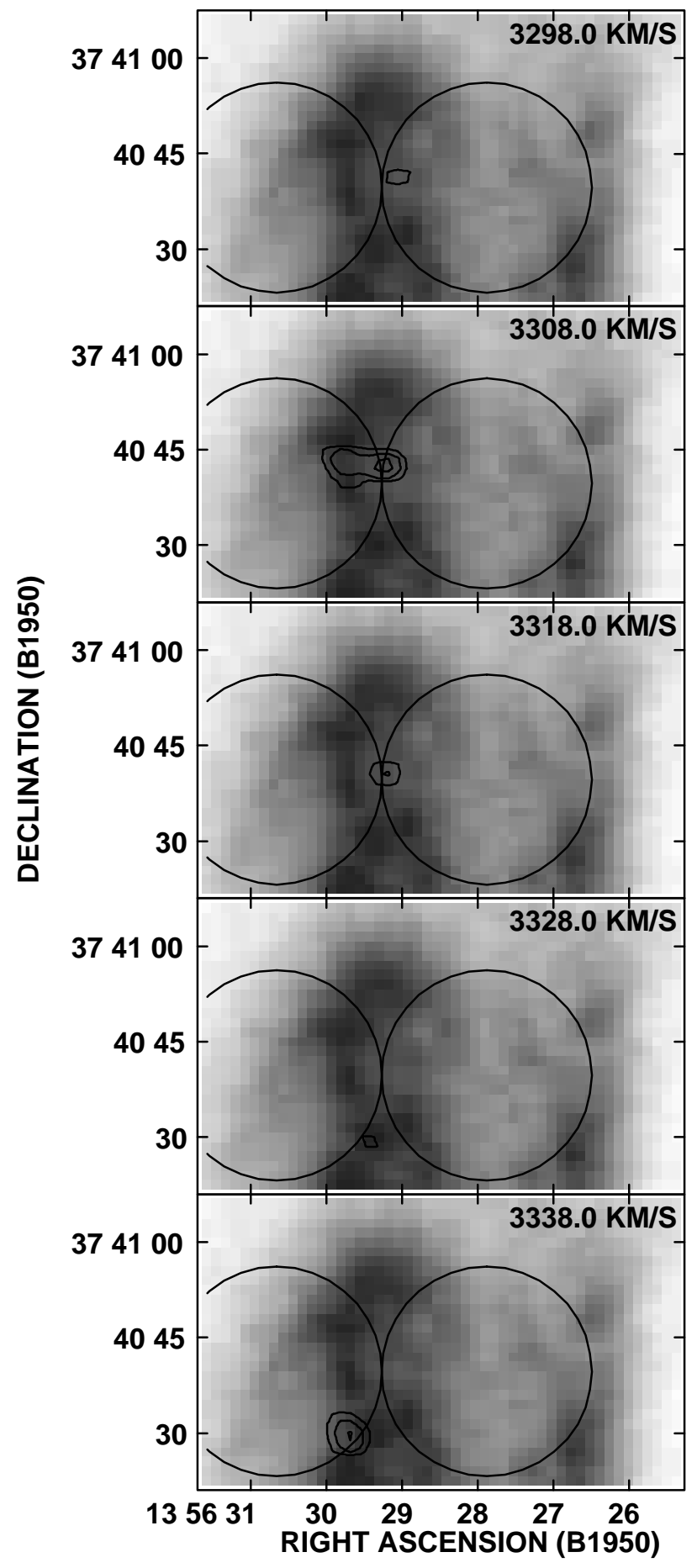

FIG. 14.-Selection of channel maps from the BIMA CO cube overlaid on a gray-scale display of the Digitized Sky Survey image of the northern part of NGC 5395. The channel maps have been masked so that only detections or possible detections are displayed. The contour levels are at 2, 3, and 4 times the rms noise. The two large circles denote Onsala positions $\mathrm{H}$ and I, labelled in Fig. 1.

Zhu et al. (1999) found an $S_{\mathrm{CO}}$ of $166 \pm 17 \mathrm{Jy} \mathrm{km} \mathrm{s}^{-1}$ (380 $\mathrm{km} \mathrm{s}^{-1} \mathrm{FWHM}$ ) from the central NRAO $12 \mathrm{~m}$ pointing, ${ }^{14}$ but neither Onsala nor BIMA detect $\mathrm{CO}$ emission from the nuclear region of NGC 5395. The discrepancy between the

14 M. Zhu (2001, private communication) points out that the value of the peak $T_{\mathrm{mb}}$ was misprinted in their Table $2 \mathrm{~A}$ as $31.8 \mathrm{mK}$ instead of 18.3 $\mathrm{mK}$.
Onsala and NRAO $12 \mathrm{~m}$ results at this location can be explained if most of the central $\mathrm{CO}$ emission seen by the NRAO $12 \mathrm{~m}$ comes from the dominant spiral arm on the eastern side of NGC 5395 (not the nucleus) and fills the NRAO beamwidth parallel to the major axis of the galaxy.

\section{CONCLUSIONS}

The spiral galaxy NGC 5394 has suffered a recent prograde grazing encounter with the spiral galaxy NGC 5395 (Paper I). In NGC 5394 we find (1) 80\% of the CO emission detected by BIMA comes from the central 800 pc starburst region, (2) the gas distribution in the disk is lopsided, with more $\mathrm{CO}, \mathrm{H} \alpha$, and $\mathrm{H}$ I emission from the western or southwestern side, and (3) $\mathrm{CO}$ and $\mathrm{H} \alpha$ emission are detected from the innermost western arm but not from the optically very bright eastern or the outer-western inner-disk arms. The H I velocity dispersion in the disk is several times higher than in undisturbed spiral galaxies (see Paper I), and the $\mathrm{CO}$ velocity dispersion at the innermost western arm is somewhat higher than normal. These properties appear to result from the tidal encounter: in an encounter simulation that reproduces some of the main features of this galaxy pair, a considerable amount of gas in NGC 5394 falls into the central region early in the collision. Previous studies (Paper I) concluded that the galaxies IC 2163, NGC 2535, and NGC 5394 form three distinct stages in the development of structures in prograde grazing encounters, with NGC 5394 the most evolved interaction in this set. Of the three, only NGC 5394 has a central starburst with a high concentration of molecular gas there, which suggests, for similar types of encounters, a central starburst may not develop until near the end of the ocular phase.

Star formation in the disk of NGC 5394 follows the gas distribution. Upper limits on the $\mathrm{CO}$ integrated intensity at the eastern inner-disk arm suggest that the instability parameter $Q_{\text {gas }}$ is presently too high for significant star formation there. So the question of why there is no evidence of ongoing star formation at the eastern inner-disk arm is really the question of why the distribution of molecular gas is lopsided. This is probably the result of an asymmetry in the collisional perturbation plus the gas losing angular momentum in shocks and may be related to the west-versuseast time lag in the development of the ocular oval in the model.

Kaufman et al. (1997) noted that widespread, high-velocity dispersion in the $\mathrm{H}$ I gas is observed in the disks of a number of interacting spiral galaxies during an early phase of post-encounter evolution. With the BIMA observations of NGC 5394 we have some evidence that enhanced turbulence in the disk is also present in the molecular gas. The measured component of the velocity dispersion in NGC 5394 should represent mainly the $z$ component. In NGC 5395 the broad CO line widths in the NRAO $12 \mathrm{~m}$ observations by Zhu et al. (1999) indicate high-velocity dispersions similar to those in the $\mathrm{H}$ I gas. Although molecular gas with highvelocity dispersion has been found previously in merger remnants and in the nuclear regions of normal galaxies (Casoli et al. 1991; Bally et al. 1988), our results indicate that molecular gas with high-velocity dispersion may also occur in the disks of galaxies involved in a grazing encounter.

If standard values are used for the conversion factor $X=N\left(\mathrm{H}_{2}\right) / I_{\mathrm{CO}}$ and the dust-to-gas ratio, then the average extinction of the NGC 5394 starburst implied by the inte- 
grated CO intensity $I_{\mathrm{CO}}$ is a factor of 3-4 greater than that deduced from a comparison of the radio continuum, $\mathrm{H} \alpha$, and $60 \mu \mathrm{m}$ luminosities. From this we conclude that the value of $X$ in the starburst is a factor of 3-4 below the standard value. This adds the starburst region in NGC 5394 to the list of nuclear starbursts in which the value of $X$ is below the standard value. The $\mathrm{CO}$ position-velocity diagram of the nucleus of NGC 5394 reveals two separate velocity features very close to the center. This may indicate a nuclear ring or the "twin peaks" of an inner ILR or some destruction of $\mathrm{CO}$ at the nucleus by photodissociation or consumption in star formation.

Very little of the CO emission detected from NGC 5395 in single-dish observations is seen in the BIMA data, and thus the molecular gas does not appear to be strongly concentrated in compressed ridges. We are left with the puzzle that on the eastern side of NGC 5395, the ring/pseudoring is brighter in radio-continuum and $\mathrm{H} \alpha$ emission, whereas on the western side of NGC 5395, it has a higher column density of total gas.

This research has made use of the NASA/IPAC Extragalactic Database (NED) which is operated by the Jet Propulsion Laboratory, California Institute of Technology, under contract with the National Aeronautics and Space Administration. We thank Tyler Nordgren for providing the fits version of his $r$-band image.

\section{REFERENCES}

Aalto, S., Booth, R. S., Black, J. H., \& Johansson, L. E. B. 1995, A\&A, 300, 369

Aalto, S., Radford, S. J. E., Scoville, N. Z., \& Sargent, A. I. 1997, ApJ, 475, L107

Adler, D. S., Allen, R. J., \& Lo, K. Y. 1991, ApJ, 382, 475

Alonso-Herrero, A., Engelbracht, C. W., Rieke, M. J., Rieke, G. H., \& Quillen, A. C. 2001, ApJ, 546, 952

Amram, P., Marcelin, M., Boulesteix, J., \& Le Coarer, E. 1989, A\&AS, 81, 59

Arp, H. 1969, A\&A, 3, 418

Athanassoula, E. 1992a, MNRAS, 259, 328 1992b, MNRAS, 259, 345

Bally, J. Stark, A. A., Wilson, R. W., \& Henkel, C. 1988, ApJ, 324, 223

Becker, R. H., White, R., L., \& Helfand, D. J. 1995, ApJ, 450, 559

Benedict, G. F. 1982, AJ, 87, 76

Bernlöhr, K. 1993, A\&A, 268, 25

Bohlin, R. C., Savage, B. D., \& Drake, J. F. 1978, ApJ, 224, 132

Braine, J., \& Dumke, M. 1998, A\&A, 333, 38

Brouillet, N., \& Schilke, P. 1993, A\&A, 277, 381

Bushouse, H. A., \& Stanford, S. A. 1992, ApJS, 79, 213

Casoli, F., Dupraz, C., Combes, F., \& Kazès, I. 1991, A\&A, 251, 1

Casoli, F., Sauty, S., Gerin, M., Boselli, A., Fouqué, P., Braine, J., Gavazzi,

G., Lequeux, J., \& Dickey, J. 1998, A\&A, 331, 451

Combes, F. 1991, ARA\&A, 29, 195

Condon, J. J. 1992, ARA\&A, 30, 575

Condon, J. J., Frayer, D. T., \& Broderick, J. J. 1991, AJ, 101, 362

Condon, J. J., Helou, G., Sanders, D. B., \& Soifer, B. T. 1990, ApJS, 73, 359

Dame, T. M., Hartmann, D., \& Thaddeus, P. 2001, ApJ, 547, 792

de Vaucouleurs, G., de Vaucouleurs, A., Corwin, H. G., Jr., Buta, R. J., Paturel, G., \& Fouqué, P. 1991, Third Reference Catalogue of Bright Galaxies (New York: Springer)

Elmegreen, B. G., Elmegreen, D. M., Chromey, F. R., Hasselbacher, D. A., \& Bissell, B. A. 1996, AJ, 111, 2233

Elmegreen, B. G., et al. 2000, AJ, 120, 630

Elmegreen, B. G., Sundin, M., Kaufman, M., Brinks, E., \& Elmegreen, D. M. 1995a, ApJ, 453, 139

Elmegreen, D. M., Kaufman, M., Brinks, E., Elmegreen, B. G., \& Sundin, M. 1995b, ApJ, 453, 100

Elmegreen, D. M., Kaufman, M., Elmegreen, B. G., Brinks, E., Struck, C.,

Klaric, M., \& Thomasson, M. 2001, AJ, 121, 182

Golla, G., Allen, M. L., \& Kronberg, P. P. 1996, ApJ, 473, 244

Horellou, C., \& Booth, R. S. 1997, A\&AS, 126, 3

Karachentsev, I. D. 1980, ApJS, 44, 137

Kaufman, M., Brinks, E., Elmegreen, B. G., Elmegreen, D. M., Klarić, M., Struck, C., Thomasson, M., \& Vogel, S. 1999a, AJ, 118, 1577 (Paper I)

Kaufman, M., Brinks, E., Elmegreen, D. M., Thomasson, M., Elmegreen, B. G., Struck, C. \& Klarić, M. 1997, AJ, 114, 2323

Kaufman, M. J., Wolfire, M. G., Hollenbach, D. J., \& Luhman 1999b, ApJ, 527, 795

Keel, W. C., Kennicutt, R. C., Jr., Hummel, E., \& van der Hulst, J. M. 1985, AJ, 90, 708

Kennicutt, R. C., Jr. 1989, ApJ, 344, 685 1998, ARA\&A, 36, 189

Leech, K. J., Penston, M. V., Terlevich, R., Lawrence, A., RowanRobinson, M., \& Crawford, J. 1989, MNRAS, 240, 349

Lonsdale-Persson, C. J., \& Helou, G. 1987, ApJ, 314, 513

Martin, C. L., \& Kennicutt, R. C., Jr. 2001, ApJ, 555, 301

Nakai, N., Hayashi, M., Handa, T., Sofue, Y., \& Hasegawa, T. 1987, PASJ, 39,685

Nordgren, T. E., Chengalur, J. N., Salpeter, E. E., \& Terzian, Y. 1997, AJ, 114,77

Regan, M. W. 2000, ApJ, 541, 142

Rieke, G. H., Loken, K., Rieke, M. J., \& Tamblyn, P. 1993, ApJ, 412, 99

Sandage, A., \& Bedke, J. 1988, Atlas of Galaxies (Washington: NASA)

Sault, R. J., Teuben, P. J., \& Wright, M. C. H. 1995, in ASP Conf. Ser. 77, Astronomical Data Analysis Software and Systems IV, ed., R. A. Shaw, H. E. Payne, \& J. J. E. Jayes (San Francisco: ASP), 433

Seaquist, E. R., \& Clark, J. 2001, ApJ, 552, 133

Shen, J., \& Lo, K. Y. 1995, ApJ, 445, L99

Shier, L. M., Rieke, M. J., \& Rieke, G. H. 1994, ApJ, 433, L9

Shopbell, P. L., \& Bland-Hawthorn, J. 1998, ApJ, 493, 129

Smith, P. A., Brand, P. W. J. L., Mountain, C. M., Puxley, P. J., \& Nakai, N. 1991, MNRAS, 252, 6P

Sodroski, T. J., et al. 1995, ApJ, 452, 262

Steer, D. G., Dewdney, P. E., \& Ito, M. R. 1984, A\&A, 137, 159

Strong, A. W., Bloemen, J. B. M., Dame, T. M., Grenier, I. A., Hermsen, W., Lebrun, F., Nyman, L.Å, Pollock, A. M. T., \& Thaddeus, P. 1988, A\&A, 207, 1

Strong, A. W., \& Mattox, J. R. 1996, A\&A, 308, L21

Thomasson, M., Sheth, K., Brinks, E., Elmegreen, B. G., Elmegreen, D. M., Kaufman, M., \& Struck, C. 2002 , in preparation

Vanzi, L., Alonso-Herrero, A., \& Rieke, G. H. 1998, ApJ, 504, 93

Wall, W. F., Jaffe, D. T., Bash, F. N., Israel, F. P., Maloney, P. R., \& Bassa, F. 1993, ApJ, 414, 98

Wild, W, Harris, A. I., Eckart, A., Genzel, R., Graf, U. U., Jackson, J. M., Russell, A. P. G., \& Stutzki, J. 1992, A\&A, 265, 447

Wong, T., \& Blitz, L. 2000, ApJ, 540, 771

Wray, J. D. 1988, The Color Atlas of Galaxies (Cambridge: Cambridge Univ. Press)

Zhu, M., Seaquist, E. R., Davoust, E., Frayer, D. T., \& Bushouse, H. A. 1999, AJ, 118, 145 